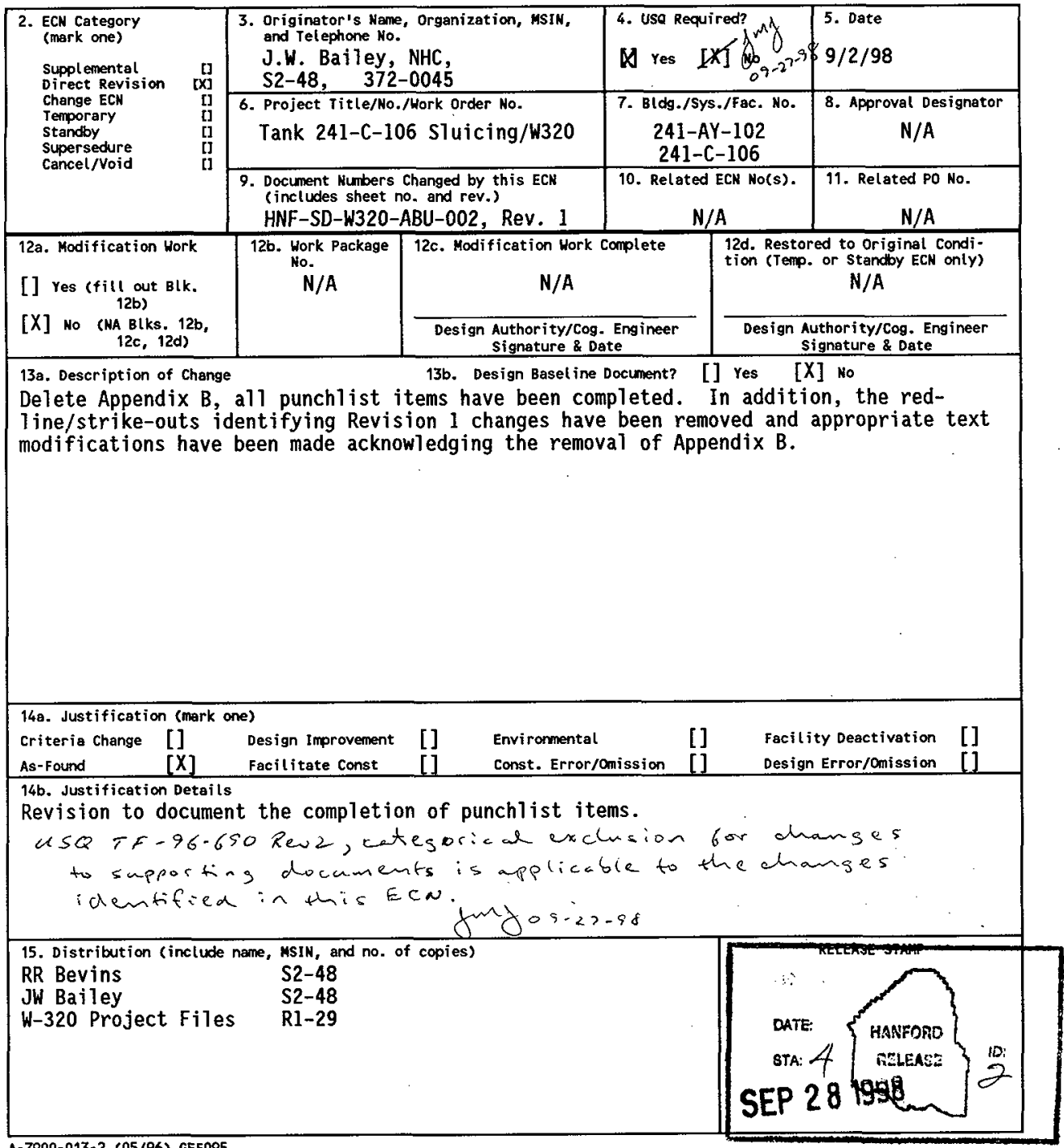




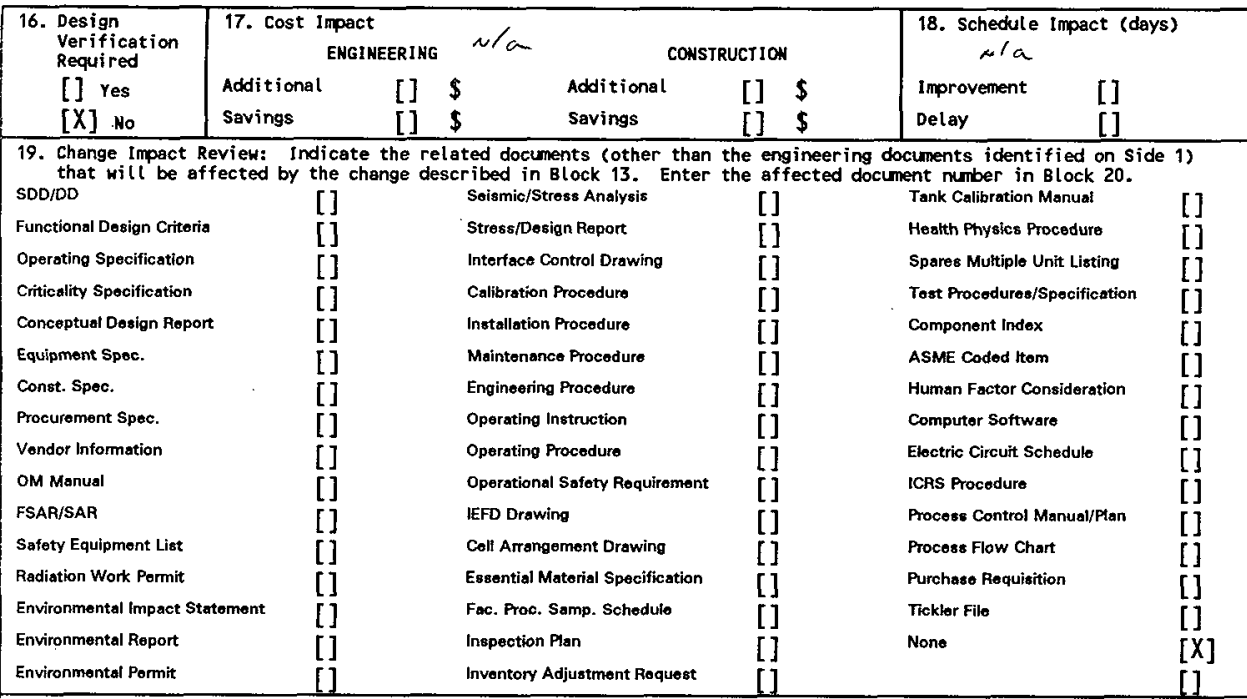

20. Other Affected Documents: (MOTE: Documents (isted below will not be revised by this ECN.) Signatures below indicate that the signing organization has been notified of other affected documents listed below. Document Number/Revision Document Number/Revision

Document Number Revision $N / A$

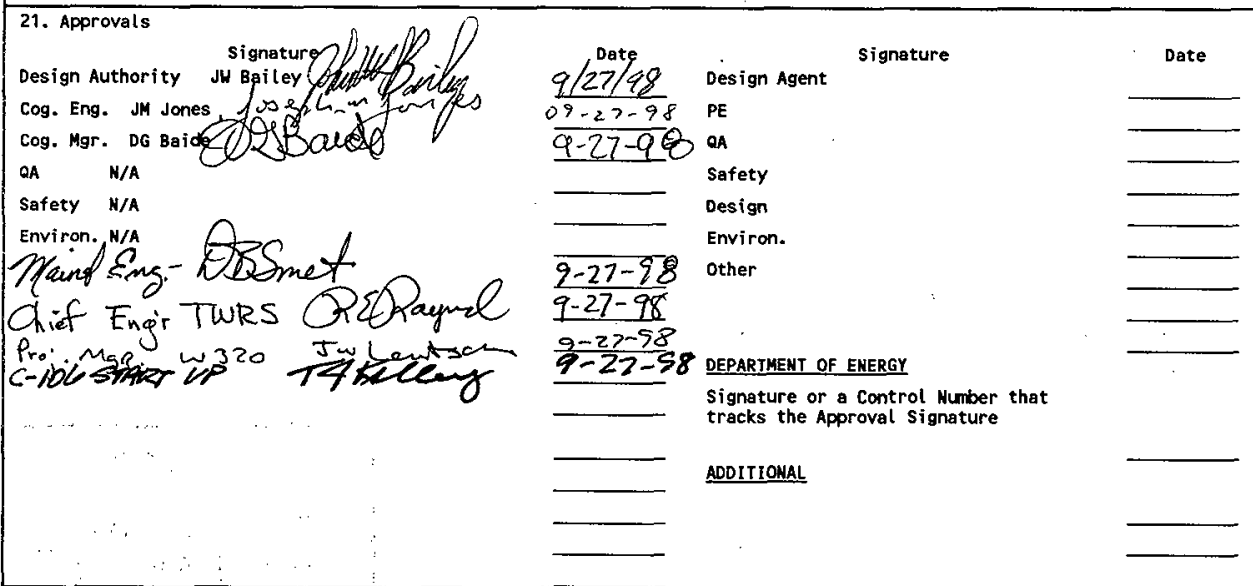




\section{Project W-320, Tank 241-C-106 Sluicing Acceptance for Beneficial Use}

J. W. Bailey

Numatec Hanford Co., Richland, WA 99352

U.S. Department of Energy Contract DE-AC09-96RLI3200

EDT/ECN: 649835

Org Code: $8 \mathrm{C} 452$

UC: 506

B\&R Code: EW3130010

Charge Code: D2991/HANA0600

Total Pages: 57

Key Words: Project $W-320$, S1uicing, Tank 241-C-106, Tank 241-AY-102, Acceptance for Beneficial Use, ABU, roadmap, WRSS.

Abstract: The purpose of this document is to identify the Project $W-320$ Chiller Documentation required to be turned over from the Projects Organization to Tank Farm Operations as part of the acceptance of the new equipment for beneficial use.

TRADEMARK DISCLAIMER. Reference herein to any specific comercial product, process, or service by trade name, trademark, manufacturer, or otherwise, does not necessarily constitute or imply its endorsement, recommendation, or favoring by the United States Government or any agency thereof or $i$ ts contractors or subcontractors.

Printed in the United States of America. To obtain copies of this document, contact: Document Control Services, P.O. Box 950, Mailstop H6-08, Richland WA 99352, Phone (509) 372-2420; Fax (509) 376-4989.
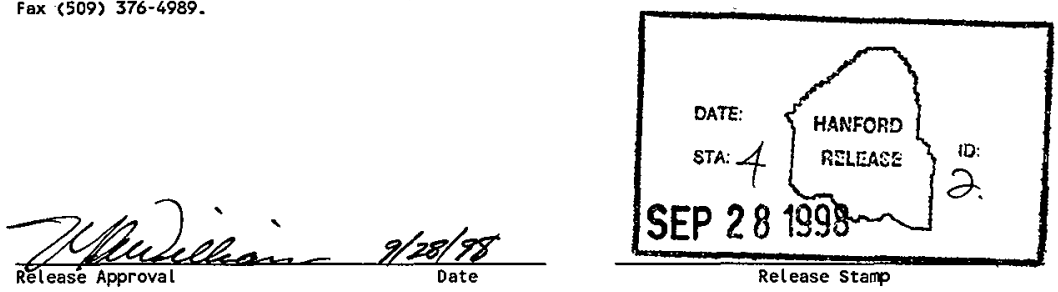

Release Stamp

\section{Approved for Public Release}


(2) ritle

Project W-320, Tank 241-C-106 Sluicing Acceptance for Beneficial Use CHANGE CONTROL RECORD

\begin{tabular}{|c|c|c|c|}
\hline \multirow{2}{*}{$\begin{array}{c}\text { (3) } \\
\text { Revision }\end{array}$} & \multirow{2}{*}{ (4) Description of Change - Replace, Add, and Delete Pages } & \multicolumn{2}{|c|}{ Authorized for Release } \\
\hline & & (5) Cog. Engr. & (6) Cog. Mgr. \\
\hline 0 & (7) Initial revision & $N / A$ & $N / A$ \\
\hline 1 & $\begin{array}{l}\text { ECN } 647627 \text {, Revision } 1 \text {, General document } \\
\text { revision to include specific documentation } \\
\text { requirements for turnover from the Project to } \\
\text { Operations. Update relects al1 modified and } \\
\text { new equipment. Additionally, procedure } \\
\text { numbers were updated to include new PHMC } \\
\text { procedure numbers. }\end{array}$ & N/A & N/A \\
\hline \multirow[t]{2}{*}{$\begin{array}{r}2 \\
R S\end{array}$} & $\begin{array}{l}\text { Deletes Appendix B, (which documents open } \\
\text { punchlist items) because all punchlist items } \\
\text { have been completed. Also deletes the red- } \\
\text { line/strike-outs which identified Revision } 1 \\
\text { changes. Appropriate text modifications have } \\
\text { been made acknowledging the removal of } \\
\text { Appendix B. }\end{array}$ & $\begin{array}{l}\text { RR Bevins } \\
R R \text { Bewide }\end{array}$ & 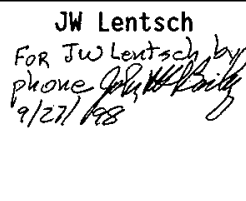 \\
\hline & $E C N-649835$ & & \\
\hline & & & \\
\hline & & & \\
\hline & & & \\
\hline & & & \\
\hline & & & \\
\hline & & & \\
\hline & & & \\
\hline & & & \\
\hline & & & \\
\hline & & & \\
\hline & & & \\
\hline & & & \\
\hline & & & \\
\hline & & & \\
\hline & & & \\
\hline & & & \\
\hline & & & \\
\hline & & & \\
\hline & & & \\
\hline & & & \\
\hline
\end{tabular}


HNF-SD-W320-ABU-002, Rev. 2

TABLE OF CONTENTS

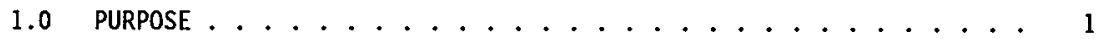

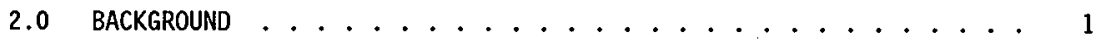

3.0 TURNOVER RESPONSIBILITIES ................ 2

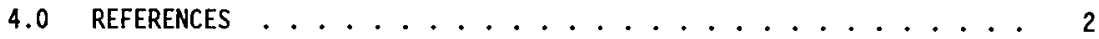

APPENDIX A DOCUMENTATION REQUIRED FOR ACCEPTANCE FOR BENEFICIAL USE . . . A-1

Table A-1: Documentation Required for Acceptance of Beneficial Use for Project W-320 Checklist . . . . . . . . . . A-3

Table A-2: Project W-320 Acceptance for Beneficial Use Required Documentation ................ A-9

ATTACHMENT I Correspondence, NHC to FDH, NHC-97571244, Project 93L-EWW320 "Tank C-106 S7uicing," DOE 6430.1A Criteria Deviation Request . AA-1

ATTACHMENT 2 Correspondence, FDH to DOE, FDH-9757289, Contract DE-ACO696RL13200, Section H-14, Laws, Regulations, and DOE Directives Deviation Request for DOE 6430.1A, "General Design Criteria" . . . AB-1

ATTACHMENT 3 Correspondence, 97-WSD-255, Contract Number DE-AC-0696RL13200 - Section H-14, Laws, Regulations and U.S. Department of Energy (DOE), Richland Operations Office (RL) Directives Deviation Request for 6430.1A "General Design Criteria" for Project W-320 Tank C-106 S7uicing . . . . . . . . . . . . AC-1 


$$
\text { HNF-SD-W320-ABU-002, Rev. } 2
$$

TITLE: "Project W-320, Tank 241-C-106 Waste Retrieval Documentation Required for Acceptance for Beneficial Use"

KEYWORDS: Project W-320, Acceptance for Beneficial Use, ABU, Tank Farm Operations, Roadmap, Structures, Systems and Components,

\subsection{PURPOSE}

The purpose of this document is to identify the Project W-320 documentation required to be turned over or be issued, readily retrievable documents from the Projects organization to Tank Farm Operations prior to acceptance of the new systems for beneficial use. The assigned responsibility for completion of each item is listed on the Acceptance for Beneficial Use (ABU) in Appendix $A$ in this document. Appendix $B$ contained a Punchlist which identifies the documentation that will be turned over following OAC II. These punchlist items have been completed and the appendix is deleted by revision 2 .

\subsection{BACKGROUND}

TWRS administrative procedure HNF-IP-0842, Volume IV, Section 3.12, Acceptance of Structures, Systems, and Components for Beneficial Use requires the project end user to identify and approve a listing of ABU requirements at the beginning of a project. At turnover, the end user accepts the structures, systems, or components (SSCs) for beneficial use based on the delivery of operable SSCS and satisfactory completion of the previously defined $A B U$ requirements.

Project W-320 was initiated prior to the implementation of this administrative procedure, therefore a listing of the $A B U$ requirements was not made at the beginning of the project. Revision 0 of this document was released in October of 1996. However, the turnover activities never commenced due to budgetary and scope concerns. Although at a late stage in the project, this document now serves as the 1 isting of $A B U$ requirements for Project $W-320$.

In addition, procedure HNF-PR0-572, Project Acceptance and Closeout was also referenced for project turnover requirements. 
HNF-SD-W320-ABU-002, Rev. 2

\subsection{TURNOVER RESPONSIBILITIES}

In general all Safety SSC design and installation requires plant (Double Shell Tank Engineering) concurrence on documentation. Comments will be generated by Double She11 Tank Engineering (DSTE) on designated documentation in Appendix A, DSTE concurrence will follow resolution of comments and inception of any required equipment modifications.

The following defines the $\mathrm{Pl}$ ant and Project responsibilities after turnover and before closeout of the ABU exceptions:

If during the exceptions closeout process SSC deficiencies (e.g. OTP failures, FDC criteria non-conformances or SEL Compliances) are identified, the Project shall correct the deficiencies.

If after turnover, SSC failures occur due to mis-operation or other causes not the responsibility of the Project, corrective actions shall be the plants responsibility.

The 0TRs; 0TR-320-007, 0TR-320-008 and 0TR-320-009 can not be completed unless and until the portable exhausters are installed. The requirement for completion of these OTRs has, therefore, been deleted from this ABU and have been incorporated into the portable exhauster deployment document, HNF-3029, Rev. 0.

The HVAC system operations which were to be contained in both WRSS Operating Procedure T0-320-008, Revision A-0, Tank 106-C HVAC System Startup/Shutdown and T0-320-010, Revision A-0, Tank C-106 WRSS HVAC System Routine Operations were instead provided in a single WRSS Operating Procedure T0-320-009, Revision A-0, Operate WRSS 296-C-006 HVAC System.

The air and water system operations which were to be contained in WRSS Operating Procedure T0-320-012, Revision A-0, Operate WRSS Air and Water System, were divided and contained in two WRSS Operating Procedures T0-320010, Operate WRSS Raw Water System, and T0-320-012, Operate WRSS Compressed Air System.

The transfer line flushing operations which were to be contained WRSS Operating Procedure T0-320-013, Revision A-0, WRSS Transfer Line flushing were provided in WRSS Operating Procedure T0-320-014, Revision A-0, Flush WRSS Transfer Lines.

The contingency transfer operations which were to be contained in WRSS Operating Procedure T0-060-051, Revision A-0, Contingency Procedure for 241AY-102 Supernate Removal were instead provided in WRSS Operating Procedure T0270-035, Revision A-0, Contingency Procedure for Routing Tank 241-AY-102 Supernate to Tank 241-AP-106. 


$$
\text { HNF-SD-W320-ABU-002, Rev. } 2
$$

The raw water system operations which were to be contained in a PCA to procedure T0-040-740, Raw Water Usage and Surveillance was instead provided in a PCA to procedure T0-040-540, Water Surveillance and Usage.

PCAs to procedures T0-020-005, Perform Pit Examinations, and T0-060-015, Monitor Breather Filters were not required.

A PCA to Alarm Response Procedure ARP-T-211-00001, Respond to Panel 3 Alarms at 271-A Control Building was not required. Al arm Response for Portable Exhausters was provided in procedures T0-060-047, T0-060-048 and T0-060-049.

A Portable Exhauster Roundsheet was provided in procedures T0-060-047, T0-060048 and T0-060-049.

Required Calibration; Preventive maintenance, functional check and functional test procedures listed in HNF-SD-W320-ABU-002, Rev. 1 have been issued.

Several items mentioned have been deleted due to requirements changes. Others have been assigned different procedure numbers than those noted in Revision 1 of the ABU.

Deleted Procedures Include:

3-GENI-372

3-GENI-376

TF-FT -359

TF-FT-259

Renumbered Procedures Include:

\begin{tabular}{lll}
\hline $6 R M-320$ & incorporated into & \\
6MISC-392 & incorporated into & TF-FT-321 \\
6-IVS-324 & became & $6-$ GENI-324 \\
6-IVS-325 & became & $6-$ GENI-325
\end{tabular}

\subsection{REFERENCES}

1. HNF-IP-0842, Volume IV, Section 3.12, Acceptance of Structures, Systems, and Components for Beneficial Use, Revision 1.

2. HNF-PRO-572, Project Acceptance and Closeout, Revision 0.

\subsection{INDEX OF ABU DOCUMENTATION}

This index is intended to serve two purposes:

- Provides a "Roadmap" to be used by Tank Farm Operations personnel of all the required turnover documentation for Project W-320.

- To document formal turnover of Project W-320 to Tank Farm Operations for beneficial use. 
HNF-SD-W320-ABU-002, Rev. 2

APPENDIX A DOCUMENTATION REQUIRED FOR ACCEPTANCE FOR BENEFICIAL USE 
HNF-SD-W320-ABU-002, Revision 2

Project Title: Project W-320 Tank C-106 Sluicing

Table A-1: Documentation Required for Acceptance of Beneficial Use for Project W-320 Checkl ist

BOCUMENTAT ION REQUIREO FOR ACCEPTANCE FOR BENET TO IAL USE

DESCRITIION

RESPONS BUE

SUPP $1 \mathrm{RB}$

ACG STANCL

AUTHORITY

ENGINEERING

[X] ABU Checklist

[X] C Farm Chiller ABU

[X] 241-AY-102 02A Pit Equipment and Modifications ABU

[X] AY-102 and C-106 Portable Exhauster ABU

[X] SHMS-C ABU

$[\mathrm{X}]$ Engineering Task $\mathrm{Plan} /$ Project Management $\mathrm{Pl}$ an

[X] Activity Schedule (Startup)

$[X]$ Basis of Interim Operation

[X] Authorization Basis Amendment Package

[X] TWRS Safety Equipment List ECN

[X] Safety Class Evaluation Document

[X] Compliance Implementation Plan

[X] BIO Compliance Matrix

[N/A] Operation Safety Document(s) - or update existing

[X] TSR Changes

[X] Thermal Analyses Related Documentation

[X] Design Criteria - (Functional Design Criteria)

\begin{tabular}{|l|l|}
\hline $\begin{array}{l}\text { NHC/JL Gilbert } \\
\text { (RR Bevins) }\end{array}$ & See ECN \\
\hline $\begin{array}{l}\text { NHC/JL Gilbert } \\
\text { (RR Bevins) }\end{array}$ & RE Raymond \\
\hline $\begin{array}{l}\text { NHC/AF Choho } \\
\text { (JW Bailey) }\end{array}$ & RE Raymond \\
\hline $\begin{array}{l}\text { NHC/AF Choho } \\
\text { (JW Bailey) }\end{array}$ & RE Raymond \\
\hline $\begin{array}{l}\text { NHC/AF Choho } \\
\text { (JW Bailey) }\end{array}$ & RE Raymond \\
\hline $\begin{array}{l}\text { NHC/JW Lentsch } \\
\text { (KB Ferlan) }\end{array}$ & RE Raymond \\
\hline $\begin{array}{l}\text { FDNW/JW Vitta } \\
\text { (MA Lane) }\end{array}$ & RE Raymond \\
\hline $\begin{array}{l}\text { DESH/CE Leach } \\
\text { (RG Stickney) }\end{array}$ & RE Raymond \\
\hline $\begin{array}{l}\text { DESH/CE Leach } \\
\text { (RG Stickney) }\end{array}$ & RE Raymond \\
\hline $\begin{array}{l}\text { NHC/JL Gilbert } \\
\text { (RR Bevins) }\end{array}$ & RE Raymond \\
\hline $\begin{array}{l}\text { NHC/JL Gilbert } \\
\text { (RR Bevins) }\end{array}$ & RE Raymond \\
\hline $\begin{array}{l}\text { DESH/CE Leach } \\
\text { (RG Stickney) }\end{array}$ & RE Raymond \\
\hline $\begin{array}{l}\text { DESH/CE Leach } \\
\text { (RG Stickney) }\end{array}$ & RE Raymond \\
\hline $\begin{array}{l}\text { NHC/AF Choho } \\
\text { (JW Bailey) }\end{array}$ & RE Raymond \\
\hline $\begin{array}{l}\text { NHC/AF Choho Leach } \\
\text { (JW Bailey) }\end{array}$ & RE Raymond \\
\hline RE Raymond \\
\hline RE
\end{tabular}




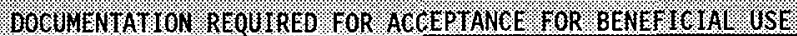

\begin{tabular}{|c|c|c|}
\hline oLschIPTION: & $\begin{array}{l}\text { RESPONSIBUE } \\
\text { SUPPLIER }\end{array}$ & 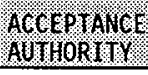 \\
\hline [X] Design Criteria - (Design Basis Document) & $\begin{array}{l}\text { NHC/AF Choho } \\
\text { (JW Bailey) }\end{array}$ & RE Raymond \\
\hline$[X]$ System Design Descriptions & $\begin{array}{l}\text { NHC/AF Choho } \\
\text { (JW Bailey) }\end{array}$ & RE Raymond \\
\hline [X] Fire Hazards Analysis & $\begin{array}{l}\text { NHC/AF Choho } \\
\text { (JW Bailey) }\end{array}$ & RE Raymond \\
\hline [X] Fire Hazards Analysis Deviation Request & $\begin{array}{l}\text { NHC/AF Choho } \\
\text { (JW Bailey) }\end{array}$ & RE Raymond \\
\hline [X] Fire Hazards Analysis Deviation Authorization & $\begin{array}{l}\text { NHC/AF Choho } \\
\text { (JW Bailey) }\end{array}$ & RE. Raymond \\
\hline$[\mathrm{X}]$ Test Plan/Specifications & $\begin{array}{l}\text { NHC/JL Gilbert } \\
\text { (RR Bevins) }\end{array}$ & RE Raymond \\
\hline [X] S/RID Compliance Assessment & $\begin{array}{l}\text { NHC } / \mathrm{JL} \text { Gilbert } \\
\text { (RR Bevins) }\end{array}$ & RE Raymond \\
\hline [X] S/RID Functional Expert Review & $\begin{array}{l}\text { NHC } / \mathrm{JL} \text { Gilbert } \\
\text { (RR Bevins) }\end{array}$ & RE Raymond \\
\hline$[X]$ Acceptance Test Procedure & $\begin{array}{l}\text { NHC } / J L \text { Gilbert } \\
\text { (RR Bevins) }\end{array}$ & RE Raymond \\
\hline [X] Acceptance (Final) Test Reports & $\begin{array}{l}\text { FDNW/JW Vitta } \\
\text { (MA Lane)/NHC } \\
\text { JL Gilbert } \\
\text { (RR Bevins) } \\
\end{array}$ & RE Raymond \\
\hline$[\mathrm{X}]$ Operational Test Procedure & $\begin{array}{l}\text { NHC/JL Gilbert } \\
\text { (RR Bevins) }\end{array}$ & RE Raymond \\
\hline [X] Operational (Final) Test Reports & $\begin{array}{l}\text { NHC/JL Gilbert } \\
\text { (RR Bevins) }\end{array}$ & RE Raymond \\
\hline [N/A] Environmental Impact Statement (See FONSI) & ----- & ---- \\
\hline$[X]$ Finding of No Significant Impact (FONSI) & $\begin{array}{l}\text { NHC/AF Choho } \\
\text { (JW Bailey) }\end{array}$ & RE Raymond \\
\hline [X] Environmental Report (Environmental Assessment) & $\begin{array}{l}\text { NHC/AF Choho } \\
\text { (JW Bailey) }\end{array}$ & RE Raymond \\
\hline [X] Environmental Permit (Notice of Construction) & $\begin{array}{l}\text { NHC/AF Choho } \\
\text { (JW Bailey) }\end{array}$ & RE Raymond \\
\hline [N/A] Hazardous Waste Disposal Plan/Procedures & ---- & 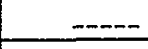 \\
\hline [N/A] Solid Waste Disposal Plan/Procedure & ---- & ---- \\
\hline [X] Waste Compatibility Studies & $\begin{array}{l}\text { NHC/AF Choho } \\
\text { (JW Bailey) }\end{array}$ & RE Raymond \\
\hline [X] Project $W-320$ Master Calculation List & $\begin{array}{l}\text { NHC/AF Choho } \\
\text { (JW Bailey) }\end{array}$ & RE Raymond \\
\hline
\end{tabular}




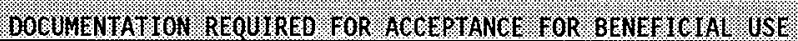

\begin{tabular}{|c|c|c|}
\hline OUSCRPPION & $\begin{array}{l}\text { RLSPONSTBL E } \\
\text { SUPPI LR }\end{array}$ & mactoraNe \\
\hline$[X]$ Calculation Notes & $\begin{array}{l}\text { NHC/AF Choho } \\
\text { (JW Bailey) }\end{array}$ & RE Raymond \\
\hline$[X]$ Stress/Seismic Analysis & $\begin{array}{l}\text { NHC/AF Choho } \\
\text { (JW Bailey) }\end{array}$ & RE Raymond \\
\hline [X] Stress/Design Report & $\begin{array}{l}\text { NHC/AF Choho } \\
\text { (JW Bailey) }\end{array}$ & RE Raymond \\
\hline$[X]$ Structural Evaluations & $\begin{array}{l}\text { NHC/AF Choho } \\
\text { (JW Bailey) }\end{array}$ & RE Raymond \\
\hline [X] Design Specifications/Report & $\begin{array}{l}\text { NHC/AF Choho } \\
\text { (JW Bailey) }\end{array}$ & RE Raymond \\
\hline [X] Equipment Specifications/Report & $\begin{array}{l}\text { NHC/AF Choho } \\
\text { (JW Bailey) }\end{array}$ & RE Raymond \\
\hline [X] Procurement Specifications & $\begin{array}{l}\text { FDNW/JW Vitta } \\
\text { (MA Lane) }\end{array}$ & RE Raymond \\
\hline$[X]$ Construction Specifications & $\begin{array}{l}\text { FDNW/JW Vitta } \\
\text { (MA Lane) }\end{array}$ & RE Raymond \\
\hline [N/A] Essential Material Specifications & ----- & ---- \\
\hline [X] Final Design Drawings & $\begin{array}{l}\text { FDNW/JW Vitta } \\
\text { (MA Lane) }\end{array}$ & RE Raymond \\
\hline [N/A] Installation Drawings & ----- & ----- \\
\hline [X] Installation Work Plan (PCPS, CWPs and JCS) & $\begin{array}{l}\text { FDNW/JW Vitta } \\
\text { (MA Lane) }\end{array}$ & RE Raymond \\
\hline $\begin{array}{l}{[X] \text { Identify Field Storage Location for PCPs and }} \\
\text { CWPS }\end{array}$ & $\begin{array}{l}\text { NHC/AF Choho } \\
\text { (JW Bailey) }\end{array}$ & RE Raymond \\
\hline$[X]$ Field Verification Plan & $\begin{array}{l}\text { NHC/AF Choho } \\
\text { (JW Bailey) }\end{array}$ & RE Raymond \\
\hline [X] Field Verified As-Built Project Drawings & $\begin{array}{l}\text { FDNW/JW Vitta } \\
\text { (MA Lane) }\end{array}$ & RE Raymond \\
\hline [N/A] Interface Control Drawings & $-\cdots-$ & ----- \\
\hline [X] Impacted Essential Facility Drawings & $\begin{array}{l}\text { FDNW/JW Vitta } \\
\text { (MA Lane) }\end{array}$ & RE Raymond \\
\hline [X] P\&ID Drawings & $\begin{array}{l}\text { FDNW/JW Vitta } \\
\text { (MA Lane) }\end{array}$ & RE Raymond \\
\hline [X] Instrument Loop Drawings & $\begin{array}{l}\text { FDNW/GP McDonald } \\
\text { (TJ Kasnick) }\end{array}$ & RE Raymond \\
\hline [N/A] Systems Drawings & $-\cdots-$ & 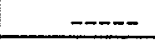 \\
\hline [N/A] Drawing Tree & $-\cdots--$ & ----- \\
\hline$[\mathrm{X}]$ Incorporate Outstanding Project Generated ECNs & $\begin{array}{l}\text { FDNW/JW Vitta } \\
\text { (MA Lane) }\end{array}$ & RE Raymond \\
\hline
\end{tabular}


DOGUMENTATION REQUIRED TOR ACCEPTANCE ROR BENEFTCIAL USE

\begin{tabular}{|c|c|c|}
\hline 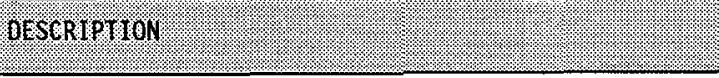 & $\begin{array}{l}\text { RESPONSIBLE } \\
\text { SUPPLIER: }\end{array}$ & 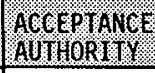 \\
\hline [X] FDNW and ICF Kaiser Calculations & $\begin{array}{l}\text { FDNW/JW Vitta } \\
\text { (MA Lane) }\end{array}$ & RE Raymond \\
\hline [X] CGI Packages & $\begin{array}{l}\text { NHC/JL Gilbert } \\
\text { (RR Bevins) }\end{array}$ & RE Raymond \\
\hline$[X]$ USQ's & $\begin{array}{l}\text { NHC/AF Choho } \\
\text { (JW Bailey) }\end{array}$ & RE Raymond \\
\hline $\begin{array}{l}{[X] \text { C-106 Stack Monitoring Work Platform }} \\
\text { Modifications Documentation }\end{array}$ & $\begin{array}{l}\text { NHC/AF Choho } \\
\text { (JW Bailey) }\end{array}$ & RE Raymond \\
\hline $\begin{array}{l}{[\mathrm{X}] \text { C-106 Process Building Area Radiation Monitor }} \\
\text { Relocation Documentation }\end{array}$ & $\begin{array}{l}\text { NHC/AF Choho } \\
\text { (JW Bailey) }\end{array}$ & RE Raymond \\
\hline $\begin{array}{l}{[X] \quad C-106 \text { Process Building Floor Drain Backflow }} \\
\text { Prevention Documentation }\end{array}$ & $\begin{array}{l}\text { NHC/AF Choho } \\
\text { (JW Bailey) }\end{array}$ & RE Raymond \\
\hline [X] C-106 Camera Installation Documentation & $\begin{array}{l}\text { NHC/AF Choho } \\
\text { (JW Bailey) }\end{array}$ & RE Raymond \\
\hline $\begin{array}{l}{[X] \text { AY-102 Temporary Camera Installation }} \\
\text { Documentation }\end{array}$ & $\begin{array}{l}\text { NHC/AF Choho } \\
\text { (JW Bailey) }\end{array}$ & RE Raymond \\
\hline [X] Project W-320 Supporting Document Bibliography & $\begin{array}{l}\text { NHC/AF Choho } \\
\text { (JW Bailey) }\end{array}$ & RE Raymond \\
\hline [X] Critical Requirements Design Review & $\begin{array}{l}\text { NHC/AF Choho } \\
\text { (JW Bailey) }\end{array}$ & RE Raymond \\
\hline [X] MOA's/MOU's & $\begin{array}{l}\text { NHC/AF Choho } \\
\text { (JW Bailey) }\end{array}$ & RE Raymond \\
\hline \multicolumn{3}{|l|}{ ADP (DAS SYSTEM) } \\
\hline [X] Software Configuration Management Plan & $\begin{array}{l}\text { FDNW/JW Vitta } \\
\text { (MA Lane) }\end{array}$ & RE Raymond \\
\hline$[X]$ Data Management $P 1$ an & $\begin{array}{l}\text { FDNW/JW Vitta } \\
\text { (MA Lane) }\end{array}$ & RE Raymond \\
\hline [N/A] System Requirements Specification & ---- & $-\cdots--$ \\
\hline$[X]$ System Description & $\begin{array}{l}\text { FDNW/JW Vitta } \\
\text { (MA Lane) }\end{array}$ & RE Raymond \\
\hline [N/A] Software Design Description & ---- & ----- \\
\hline$[X]$ Software Validation/Verification & $\begin{array}{l}\mathrm{FDNW} / \mathrm{JW} \text { Vitta } \\
\text { (MA Lane) }\end{array}$ & RE Raymond \\
\hline \multicolumn{3}{|l|}{ TRAINING } \\
\hline$[\mathrm{X}]$ Training Plan & $\begin{array}{l}\text { NHC/AF Choho } \\
\text { (JW Bailey) }\end{array}$ & TJ Kelley \\
\hline$[\mathrm{X}]$ Training Manuals & $\begin{array}{l}\text { NHC/AF Choho } \\
\text { (JW Bailey) }\end{array}$ & TJ Kelley \\
\hline
\end{tabular}




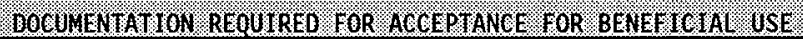

\begin{tabular}{|c|c|c|}
\hline 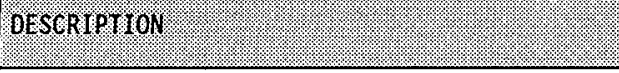 & RESPONSIBIE & 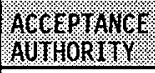 \\
\hline [X] Training to Operating Crews & $\begin{array}{l}\text { NHC/AF Choho } \\
\text { (JW Bailey) }\end{array}$ & TJ Kelley \\
\hline [X] Training to Maintenance Crews & $\begin{array}{l}\text { NHC/AF Choho } \\
\text { (JW Bailey) }\end{array}$ & DB Smet \\
\hline [X] Training Mock-up OJT & $\begin{array}{l}\text { NHC/AF Choho } \\
\text { (JW Bailey) }\end{array}$ & TJ Kelley \\
\hline [X] Vendor Supplied Training & FDNW (DAS System) & TJ Kelley \\
\hline \multicolumn{3}{|c|}{ OPERATIONS/MAINTENANCE } \\
\hline [X] Operating and Maintenance Manuals (CVI) & $\begin{array}{l}\text { NHC/AF Choho } \\
\text { (JW Bailey) }\end{array}$ & $\begin{array}{l}\text { TJ Kelley/ } \\
\text { DB Smet }\end{array}$ \\
\hline$[X]$ Process Control Plan (PCP) & $\begin{array}{l}\text { NHC/AF Choho } \\
\text { (JW Bailey) }\end{array}$ & $\begin{array}{l}\text { TJ Kelley/ } \\
\text { RE. Raymond }\end{array}$ \\
\hline$[\mathrm{X}]$ Operating Procedures. & $\begin{array}{l}\text { NHC/AF Choho } \\
\text { (JW Ba i ley) }\end{array}$ & TJ Kelley \\
\hline [X] A] arm Response & $\begin{array}{l}\text { NHC/AF Choho } \\
\text { (JW Bailey) }\end{array}$ & TJ Kelley \\
\hline [X] Surveillance Procedures & $\begin{array}{l}\text { NHC/AF Choho } \\
\text { (JW Bailey) }\end{array}$ & TJ Kelley \\
\hline [X] Calibration Procedures & $\begin{array}{l}\text { NHC/JL Gilbert } \\
\text { (RR Bevins) }\end{array}$ & DB Smet \\
\hline$[\mathrm{X}]$ Preventative Maintenance Procedures & RR Bevins & DB Smet \\
\hline [N/A] Repair/Maintenance Procedures & ----- & ----- \\
\hline [X] Functional Check Procedures & $\begin{array}{l}\text { NHC/JL Gilbert } \\
\text { (RR Bevins) }\end{array}$ & DB Smet \\
\hline [X] Functional Test Procedures & $\begin{array}{l}\text { NHC/JL Gilbert } \\
\text { (RR Bevins) }\end{array}$ & $\begin{array}{l}\text { TJ Kelley/ } \\
\text { DB Smet }\end{array}$ \\
\hline [X] Emergency Response Procedure & $\begin{array}{l}\text { NHC/AF Choho } \\
\text { (JW Bailey) }\end{array}$ & TJ Kelley \\
\hline [X] Maintenance Plan & $\begin{array}{l}\text { NHC/JL Gilbert } \\
\text { (RR Bevins) }\end{array}$ & DB Smet \\
\hline [X] PM/S Datasheets & $\begin{array}{l}\text { NHC/JL Gilbert } \\
\text { (RR Bevins) }\end{array}$ & DB Smet \\
\hline$[X]$ Instrument List & $\begin{array}{l}\text { NHC/JL Gilbert } \\
\text { (RR Bevins) }\end{array}$ & DB Smet \\
\hline [X] Setpoint Justification Document & $\begin{array}{l}\text { NHC/JL Gilbert } \\
\text { (RR Bevins) }\end{array}$ & $\begin{array}{l}\text { DB Smet/ } \\
\text { RE Raymond }\end{array}$ \\
\hline
\end{tabular}


HNF-SD-W320-ABU-002, Revision 2

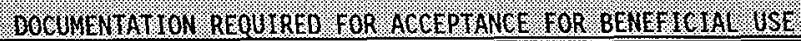

\begin{tabular}{|c|c|c|}
\hline OESERI PTION & $\begin{array}{l}\text { RESPONSIBLE } \\
\text { SUPPLIER: }\end{array}$ & $\begin{array}{l}\text { ACGEPTANer } \\
\text { AurHORMT: }\end{array}$ \\
\hline \multicolumn{3}{|c|}{ QUALITY ASSURANCE } \\
\hline$[\mathrm{X}]$ Inspection $\mathrm{Plan}$ & $\begin{array}{l}\text { FDNW/JA Peltier } \\
\text { (MA Lane) }\end{array}$ & RE Raymond \\
\hline$[X]$ QAPP (Project and TWRS) & $\begin{array}{l}\text { FDNW/JA Peltier } \\
\text { (KC Conrad) }\end{array}$ & RE Raymond \\
\hline$[N / A] Q A P, P$ & ----- & $-\cdots-$ \\
\hline \multicolumn{3}{|c|}{ PROCUREMENT } \\
\hline$[\mathrm{X}]$ Vendor Information Files & $\begin{array}{l}\text { NHC/AF Choho } \\
\text { (JW Bailey) }\end{array}$ & $\begin{array}{l}\text { DB Smet/ } \\
\text { RE Raymond } \\
\end{array}$ \\
\hline [X] Warranties & $\begin{array}{l}\text { NHC/AF Choho } \\
\text { (JW Bailey) }\end{array}$ & DB Smet \\
\hline$[\mathrm{X}]$ Master Equipment List & $\begin{array}{l}\text { NHC/JL Gilbert } \\
\text { (RR Bevins) }\end{array}$ & DB Smet \\
\hline [X] Spare Parts List & $\begin{array}{l}\text { NHC/JL Gilbert } \\
\text { (RR Bevins) }\end{array}$ & DB Smet \\
\hline [X] Spare Parts in Stock & $\begin{array}{l}\text { NHC/JL Gilbert } \\
\text { (RR Bevins) }\end{array}$ & DB Smet \\
\hline
\end{tabular}




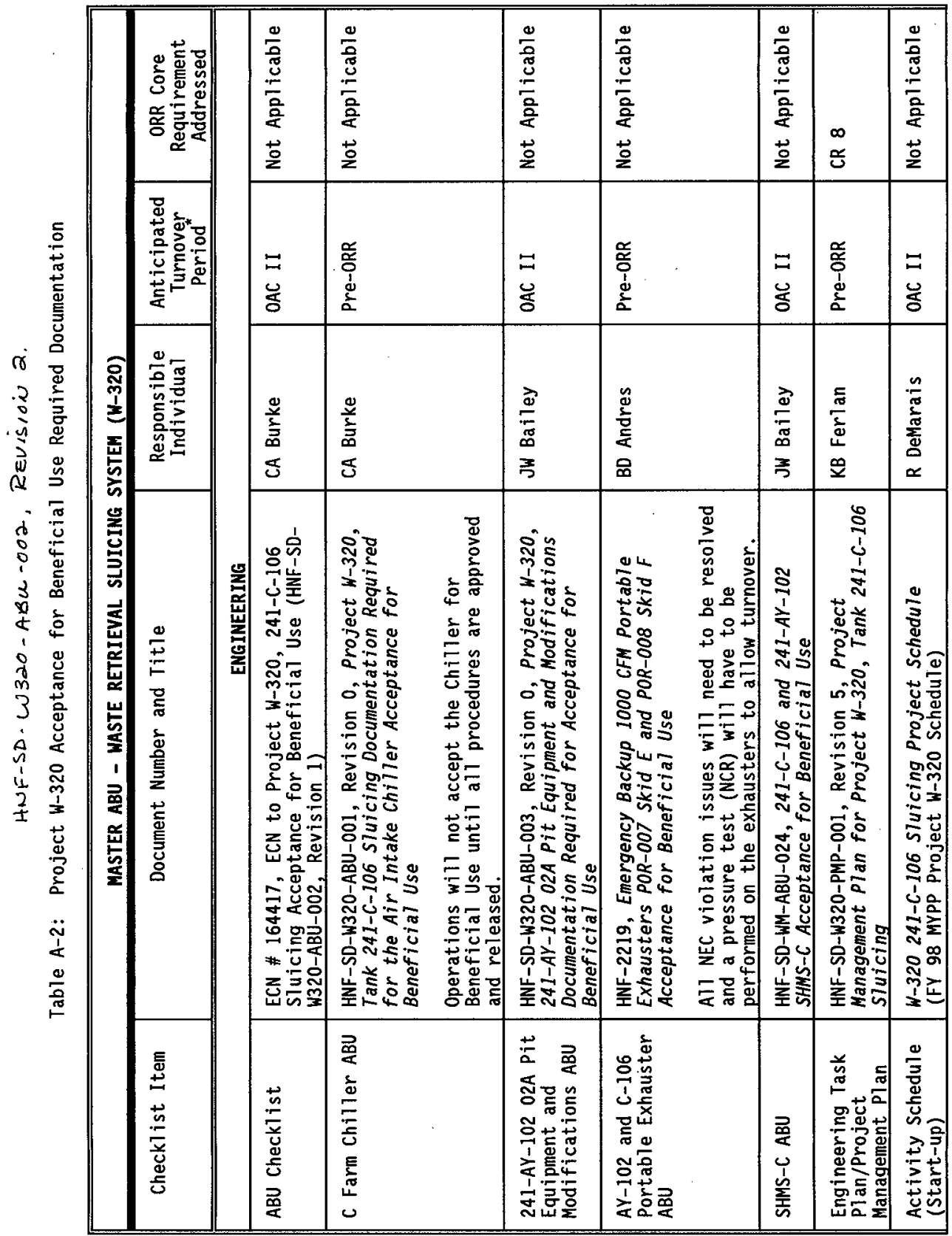




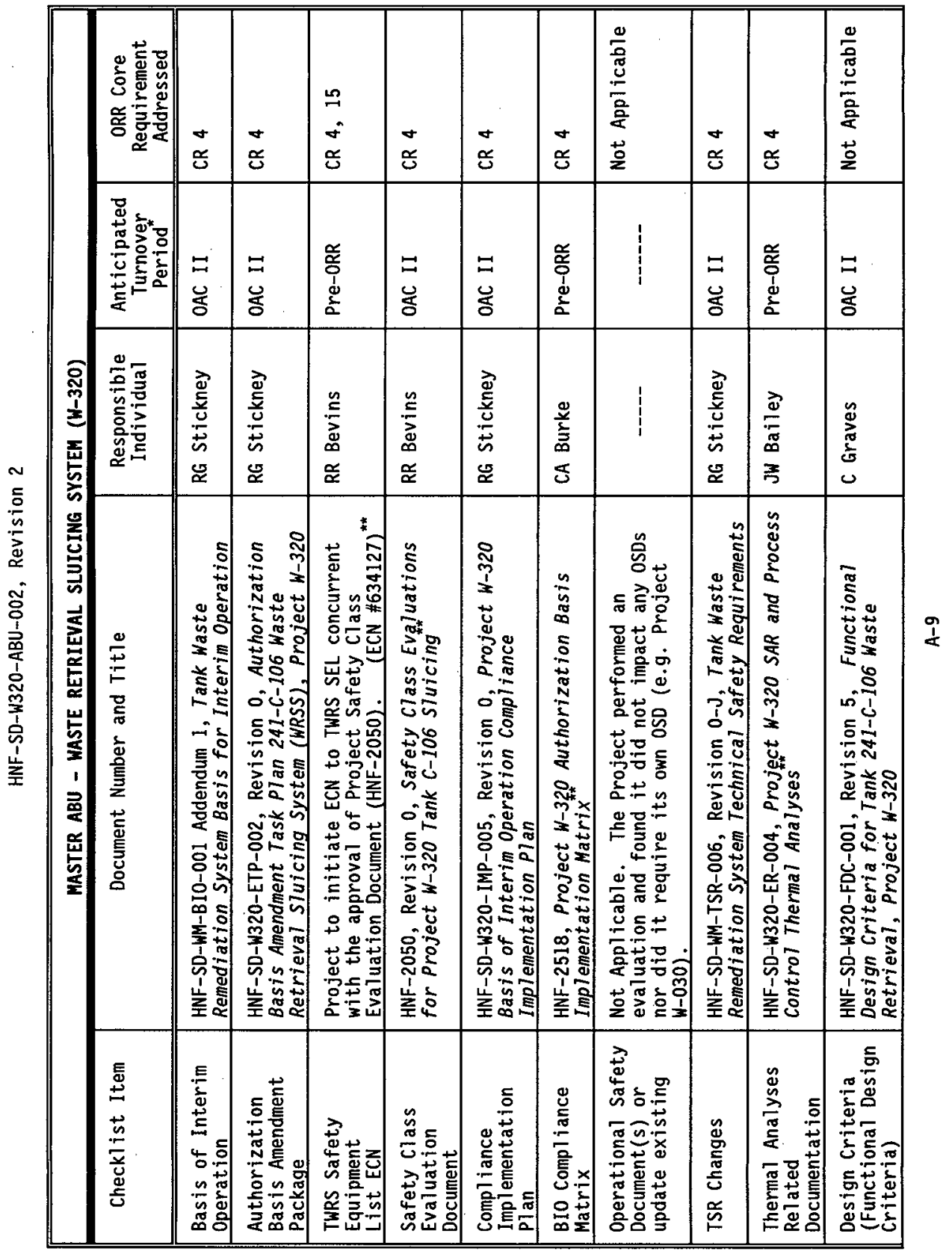




\begin{tabular}{|c|c|c|c|c|c|c|c|c|}
\hline \multirow{5}{*}{ 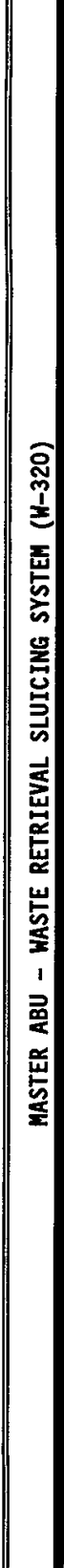 } & : & 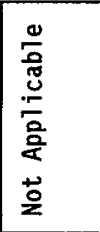 & $\begin{array}{l}\frac{0}{0} \\
\frac{0}{5} \\
\frac{0}{2} \\
\frac{2}{2} \\
+0 \\
\frac{0}{2}\end{array}$ & $\begin{array}{l}\dot{a} \\
\stackrel{ }{0}\end{array}$ & व & & $\underset{\sim}{\alpha}$ & $\begin{array}{l}\text { 음 } \\
\text { 웅 }\end{array}$ \\
\hline & 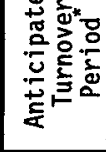 & ت & $\begin{array}{l}\Xi \\
\text { to }\end{array}$ & 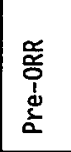 & $\begin{array}{l}\Xi \\
\text { ப̆ }\end{array}$ & & $\begin{array}{l}\Xi \\
\check{\Xi}\end{array}$ & $\begin{array}{l}\Xi \\
\ddot{y}\end{array}$ \\
\hline & 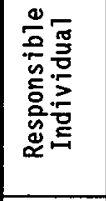 & 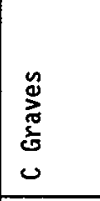 & 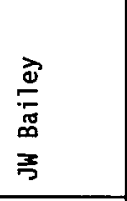 & 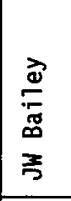 & 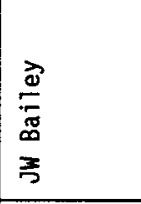 & & 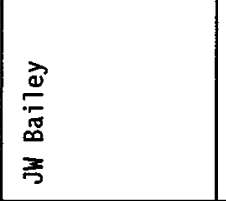 & 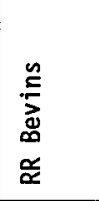 \\
\hline & 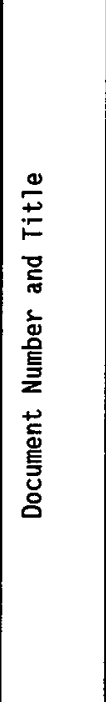 & 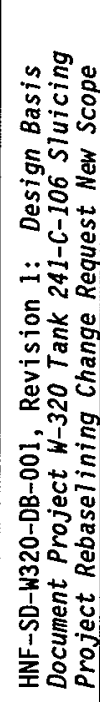 & 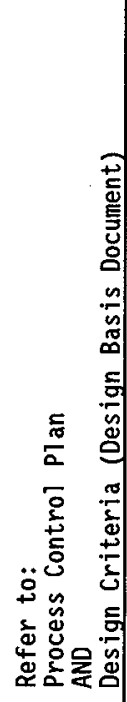 & 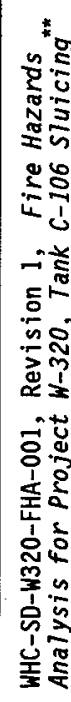 & 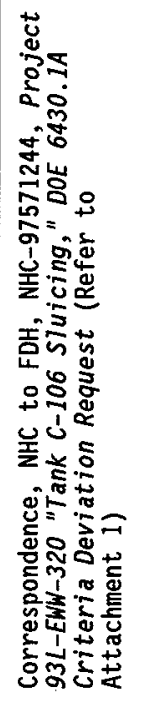 & 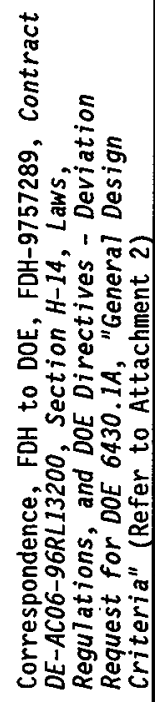 & 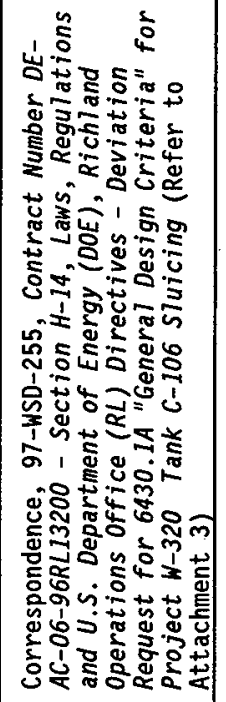 & 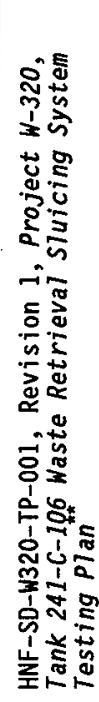 \\
\hline & 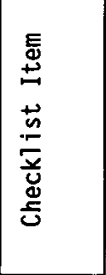 & 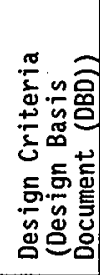 & 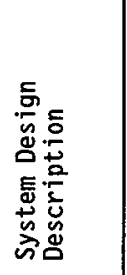 & 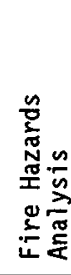 & 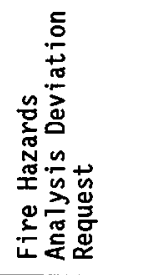 & & 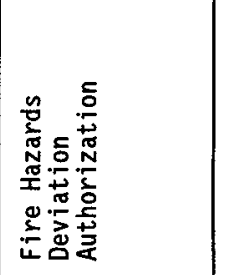 & 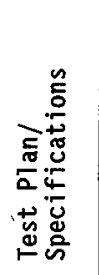 \\
\hline
\end{tabular}




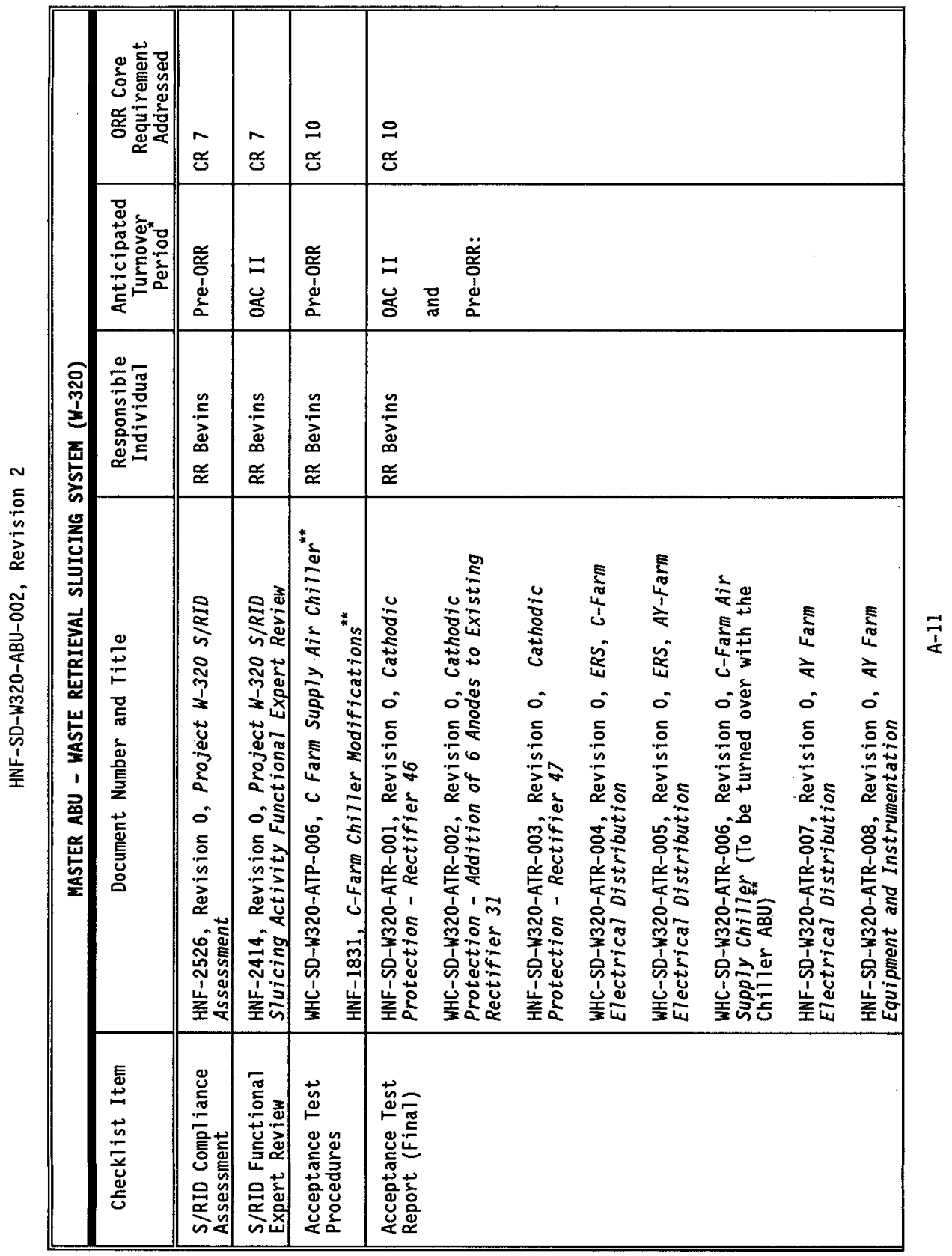




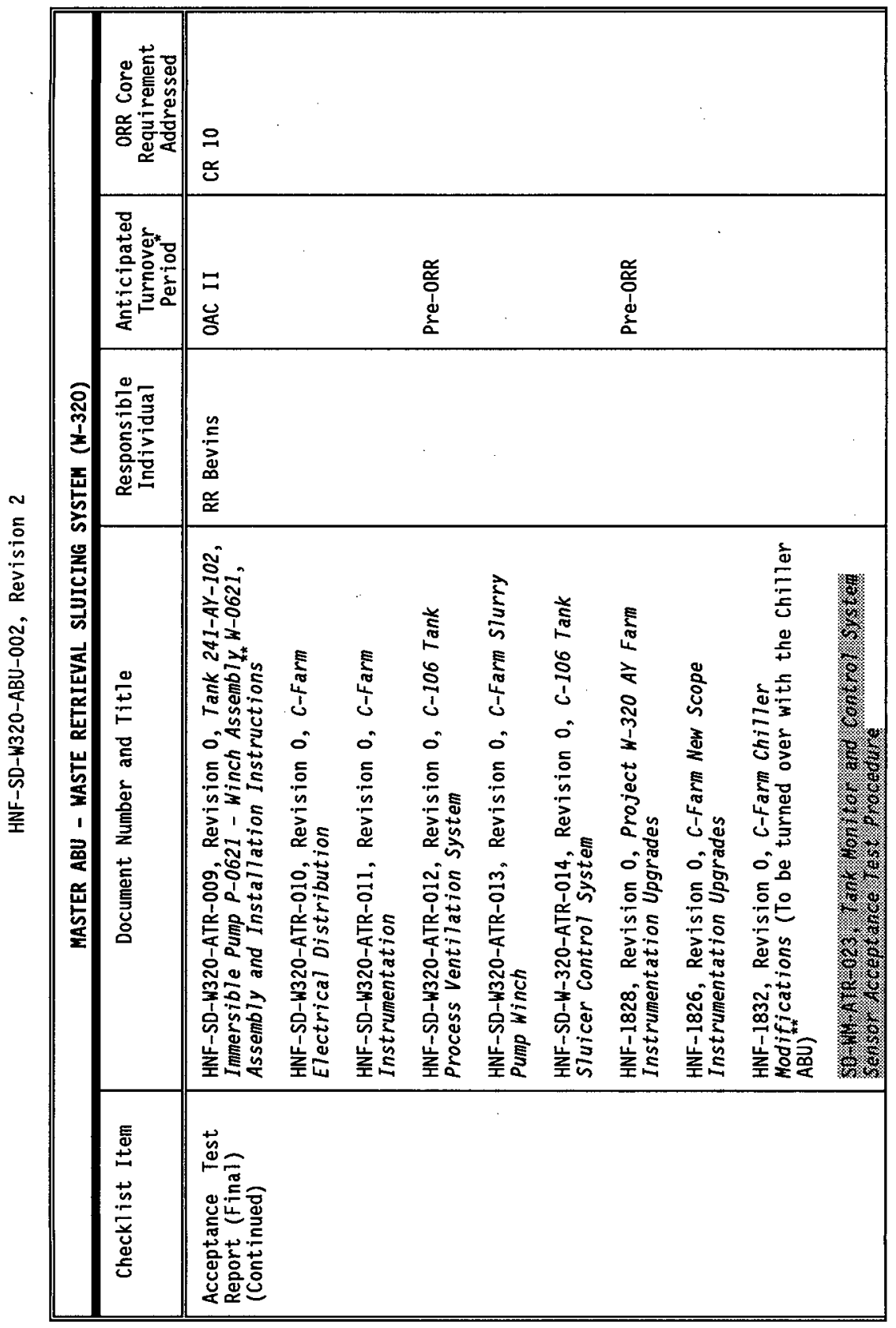




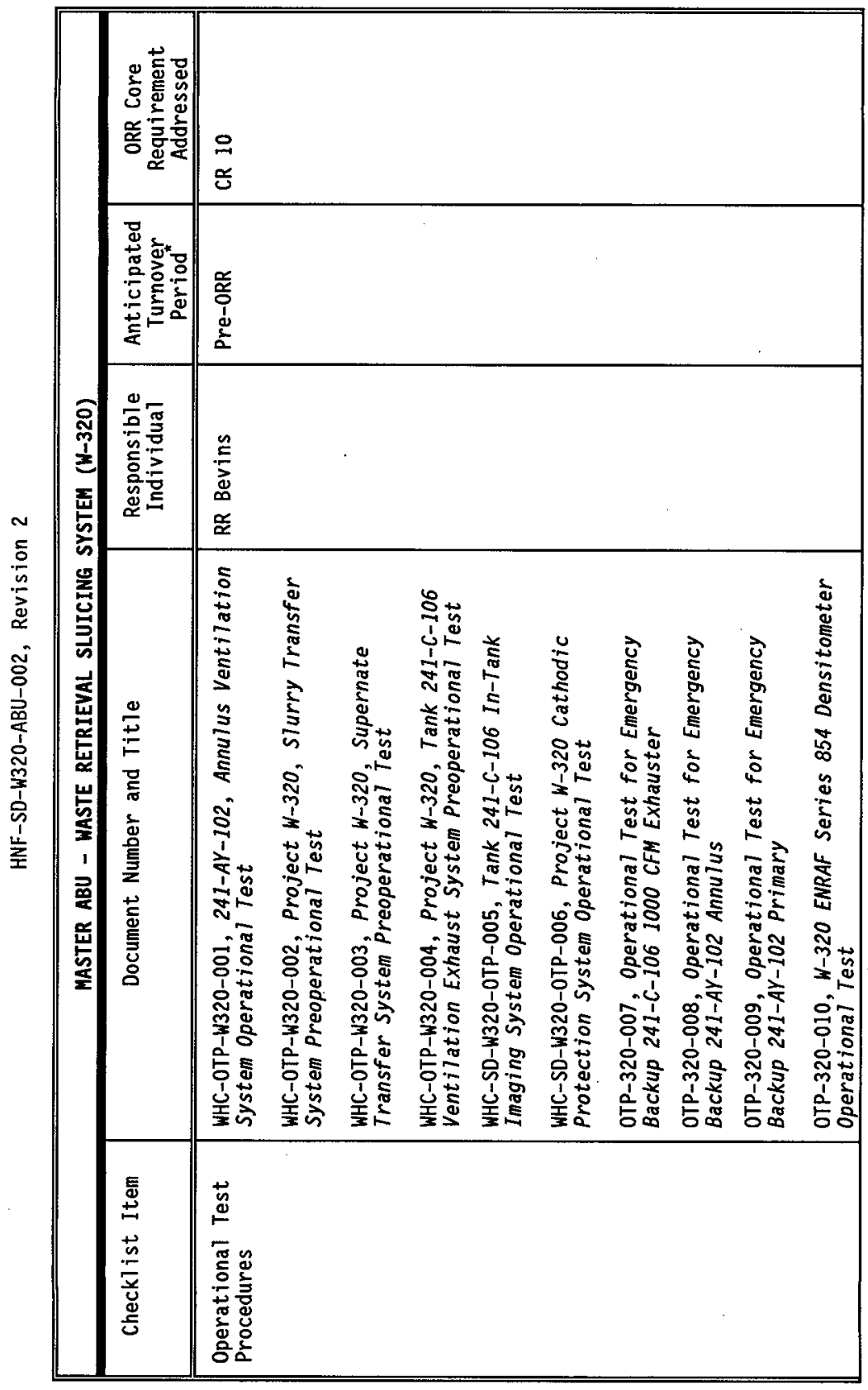




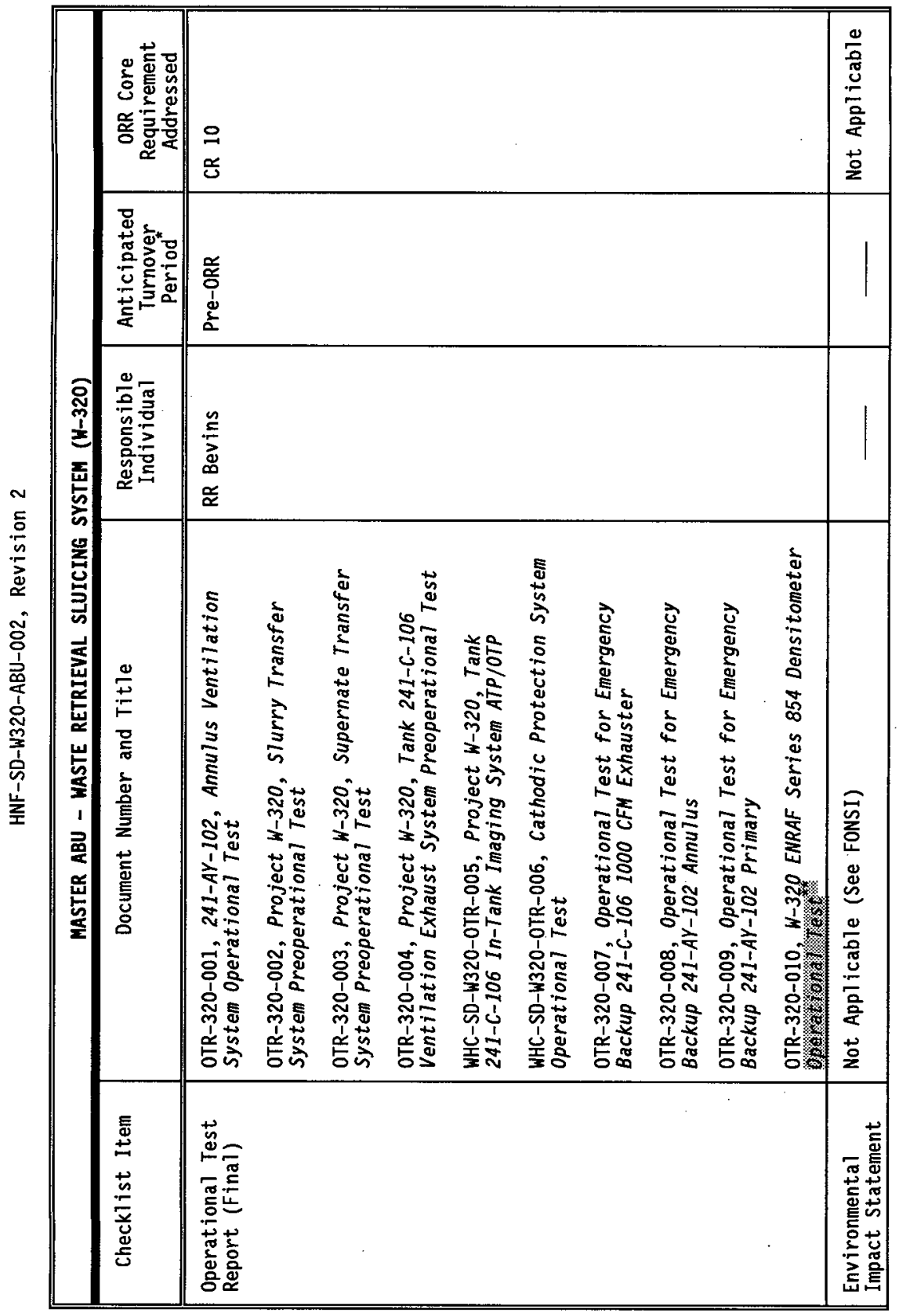




\begin{tabular}{|c|c|c|c|c|c|c|c|c|}
\hline & 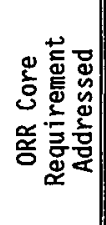 & 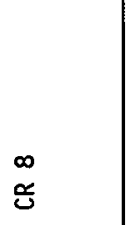 & $\begin{array}{l}\infty \\
0 \\
0\end{array}$ & $\begin{array}{l}\infty \\
\text { 品 }\end{array}$ & $\begin{array}{l}\frac{0}{0} \\
\frac{0}{0} \\
\frac{0}{0} \\
\frac{0}{2} \\
0 \\
0 \\
\frac{0}{2}\end{array}$ & 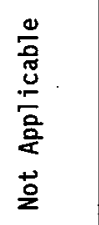 & $\begin{array}{l}\frac{0}{0} \\
\frac{0}{0} \\
\frac{0}{0} \\
\frac{0}{2} \\
\stackrel{0}{2}\end{array}$ & 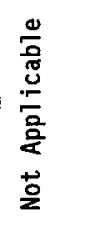 \\
\hline & 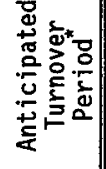 & $\begin{array}{l}\vec{~} \\
\ddot{\Xi}\end{array}$ & $\begin{array}{l}\vec{z} \\
\stackrel{\Delta}{\Delta}\end{array}$ & $\begin{array}{l}\vec{F} \\
\ddot{\Xi}\end{array}$ & $i$ & & 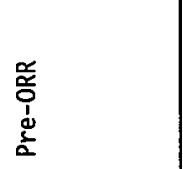 & 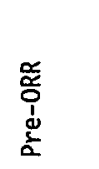 \\
\hline 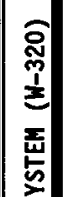 & 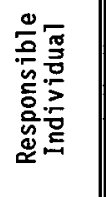 & 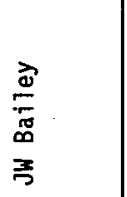 & 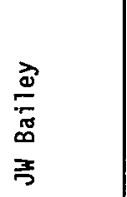 & 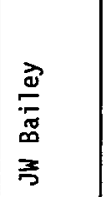 & 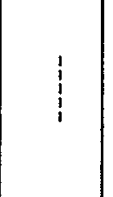 & & 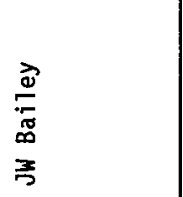 & 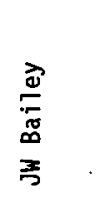 \\
\hline 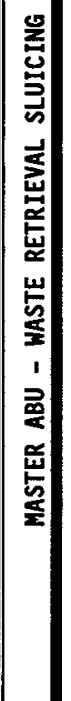 & 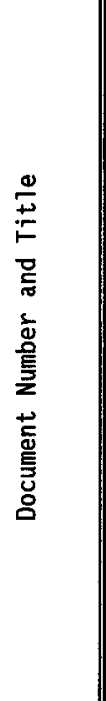 & 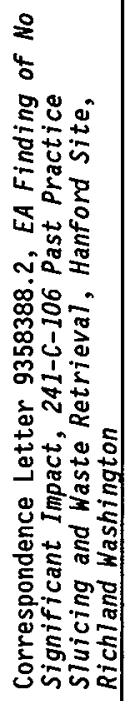 & 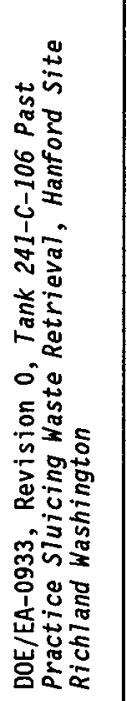 & 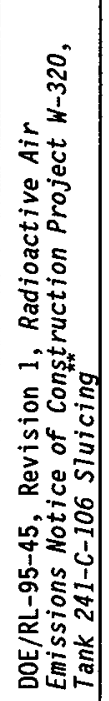 & 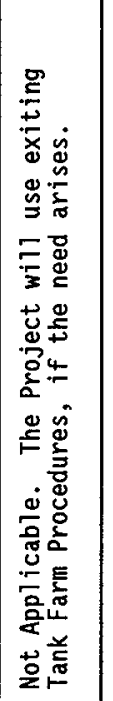 & 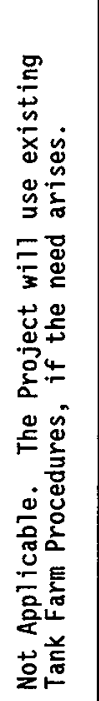 & 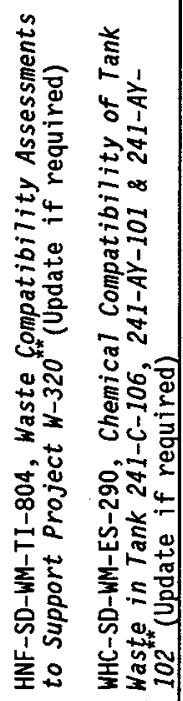 & 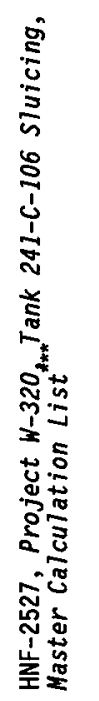 \\
\hline & 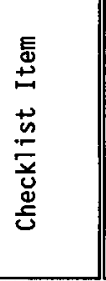 & 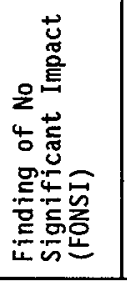 & 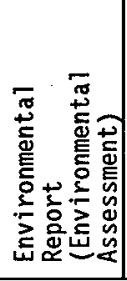 & 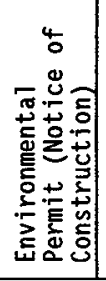 & 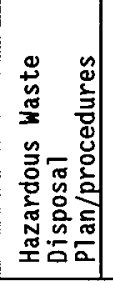 & 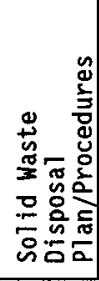 & 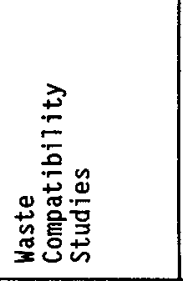 & 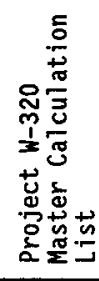 \\
\hline
\end{tabular}




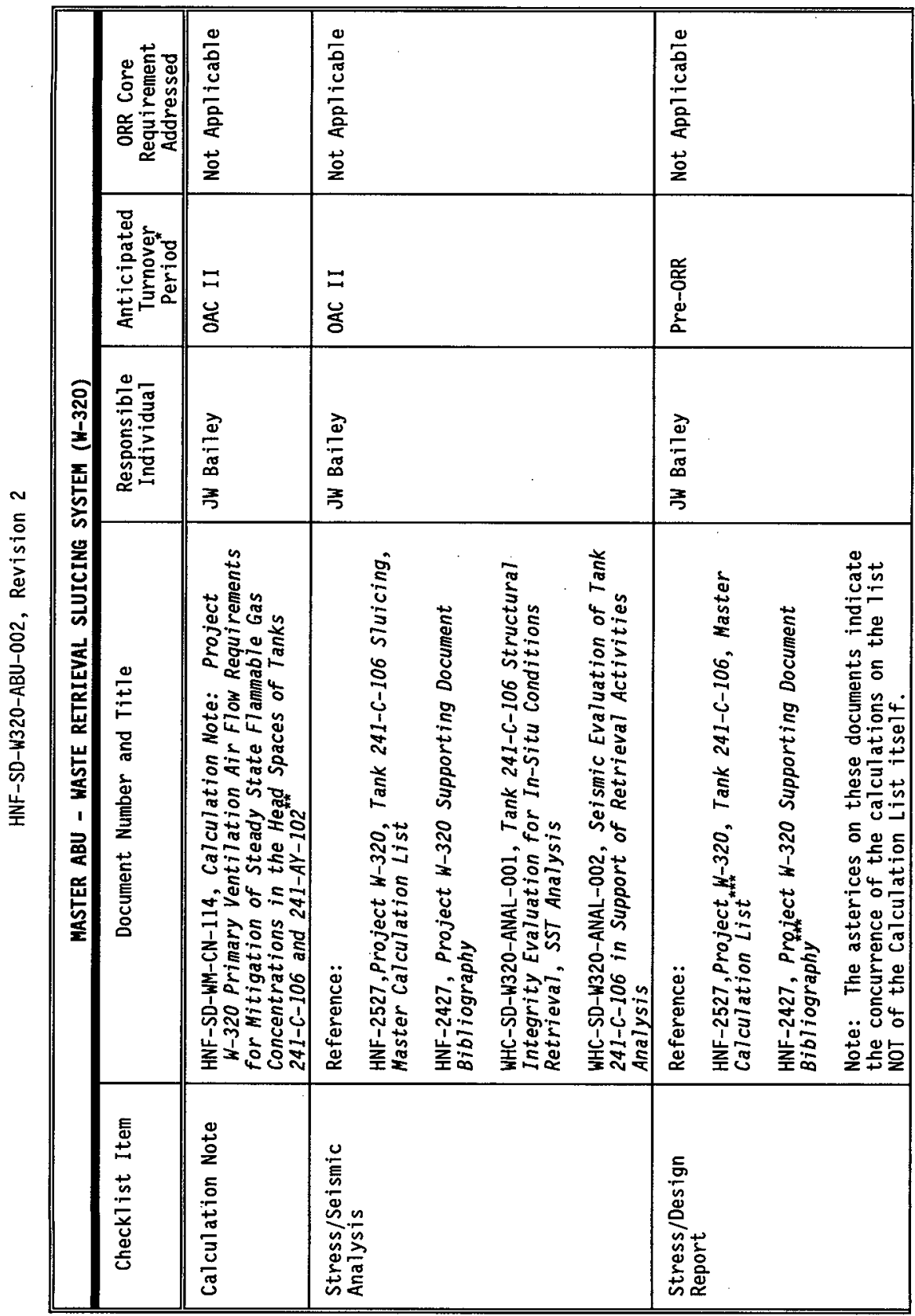




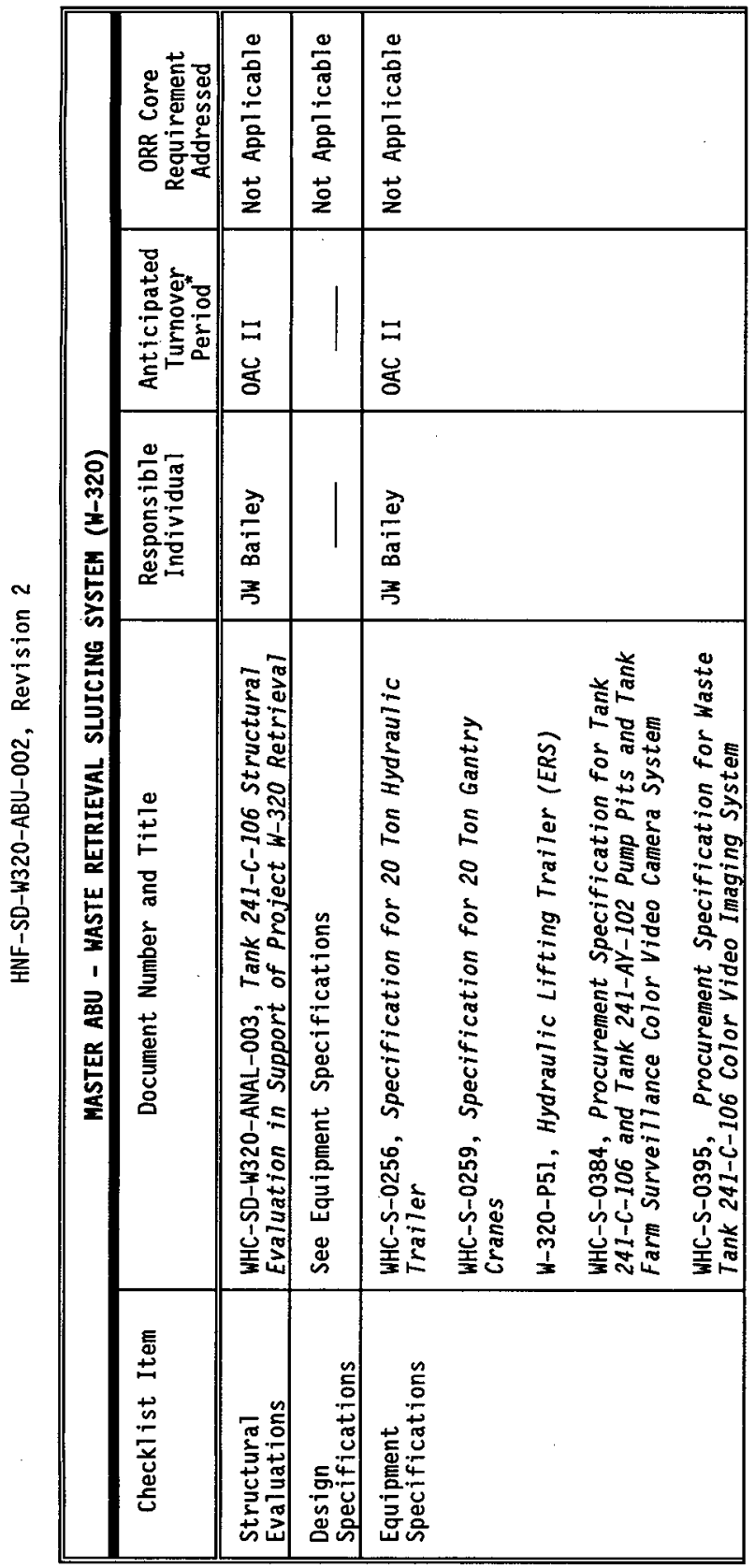




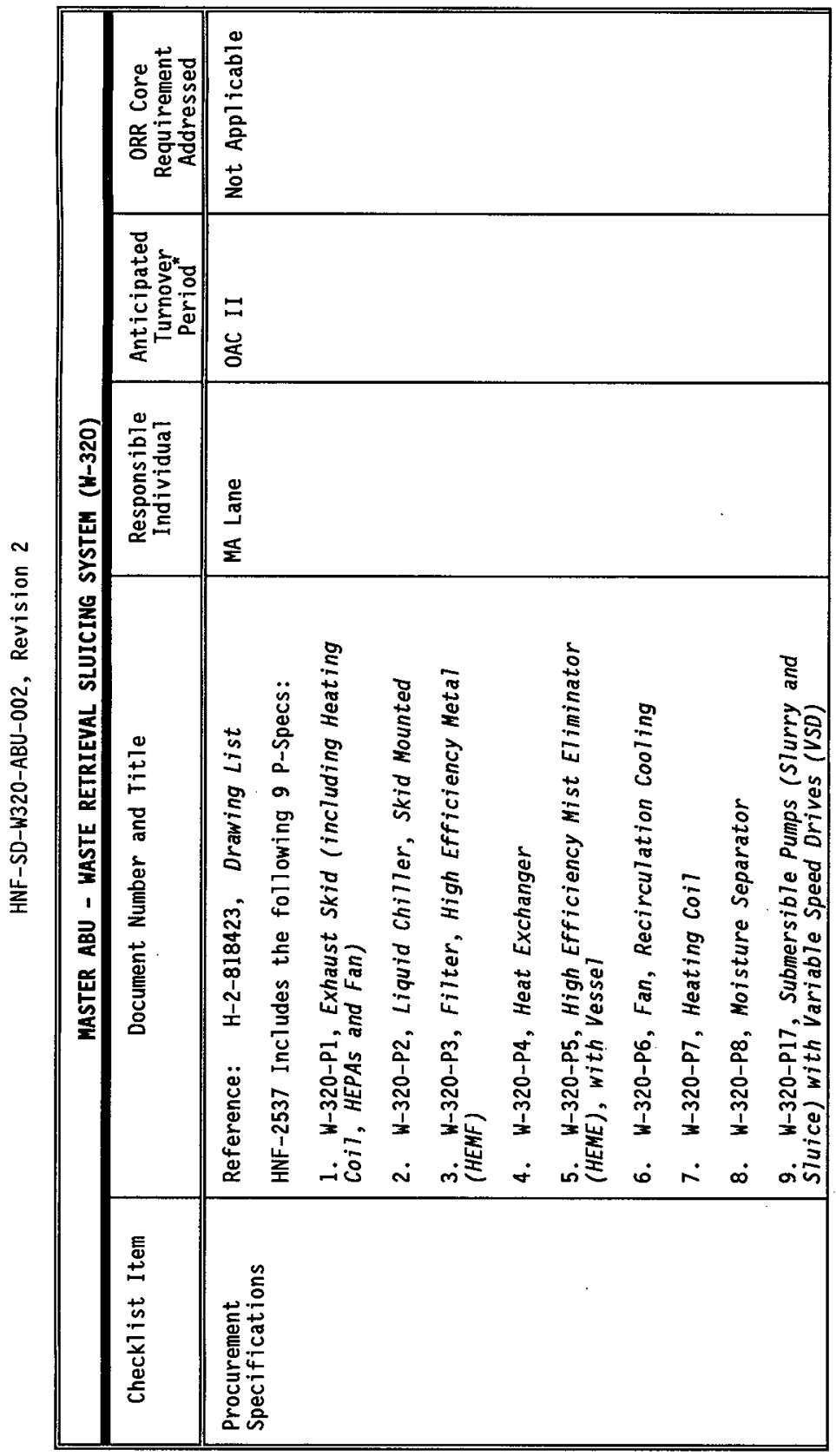




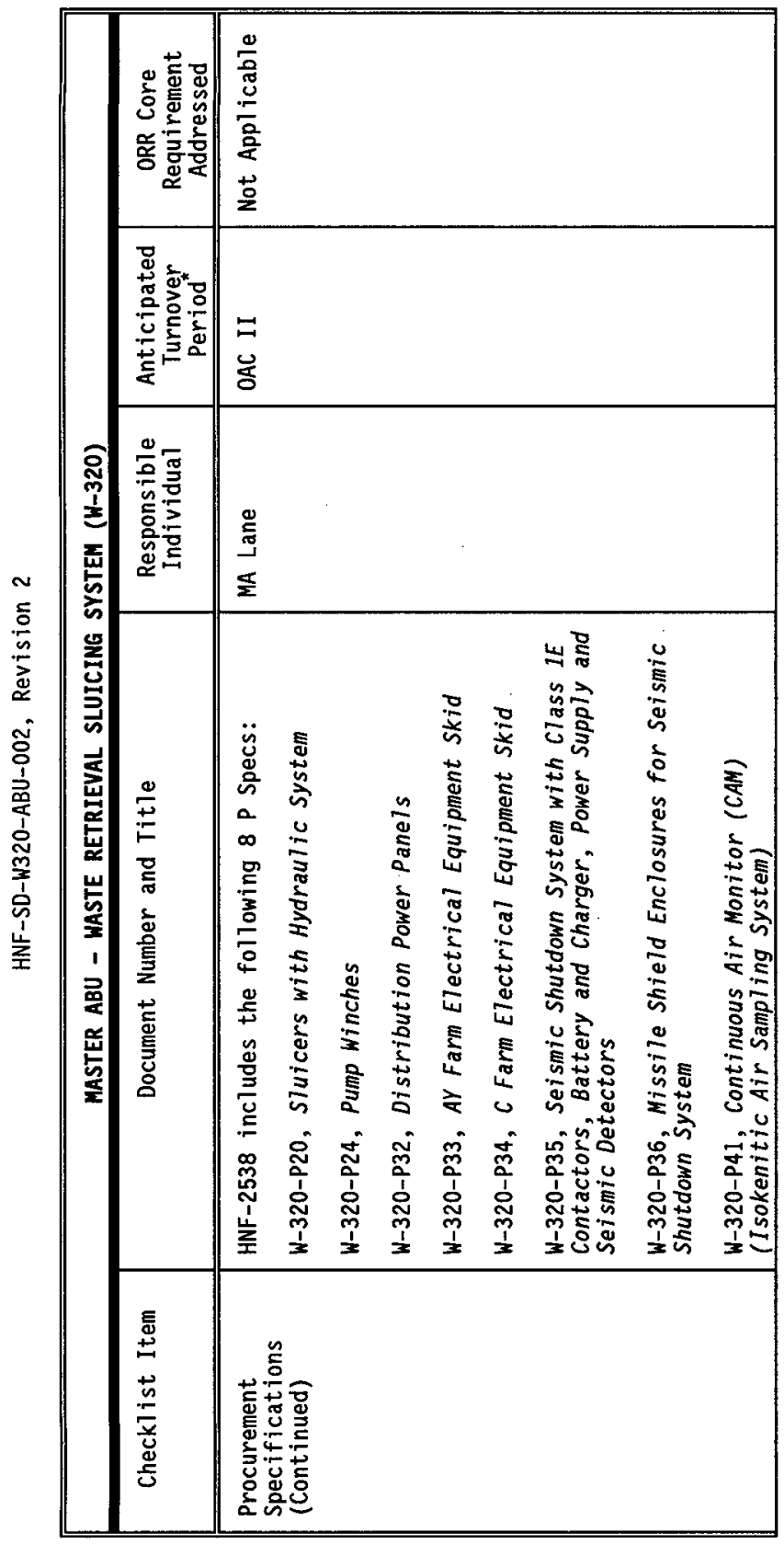




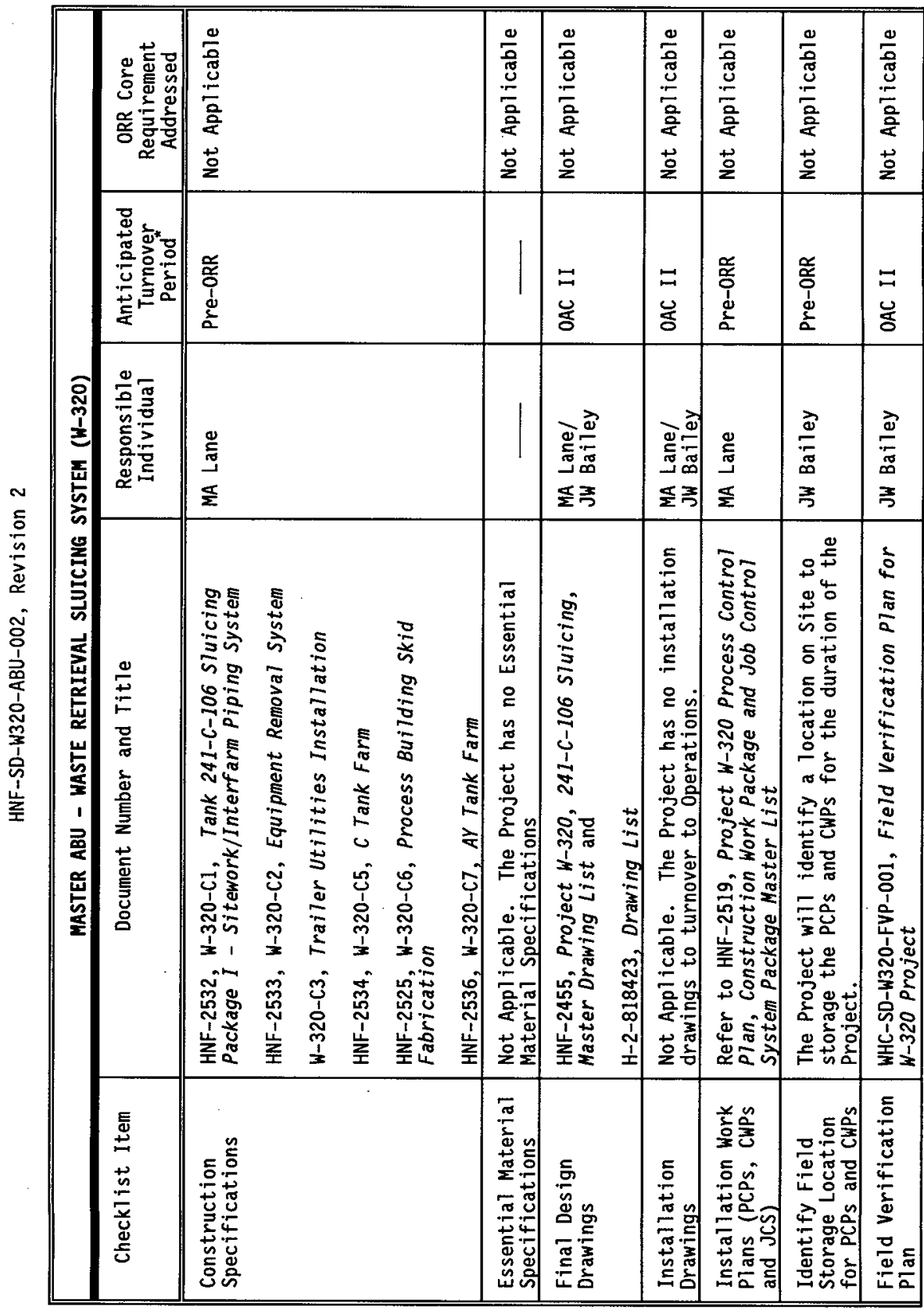




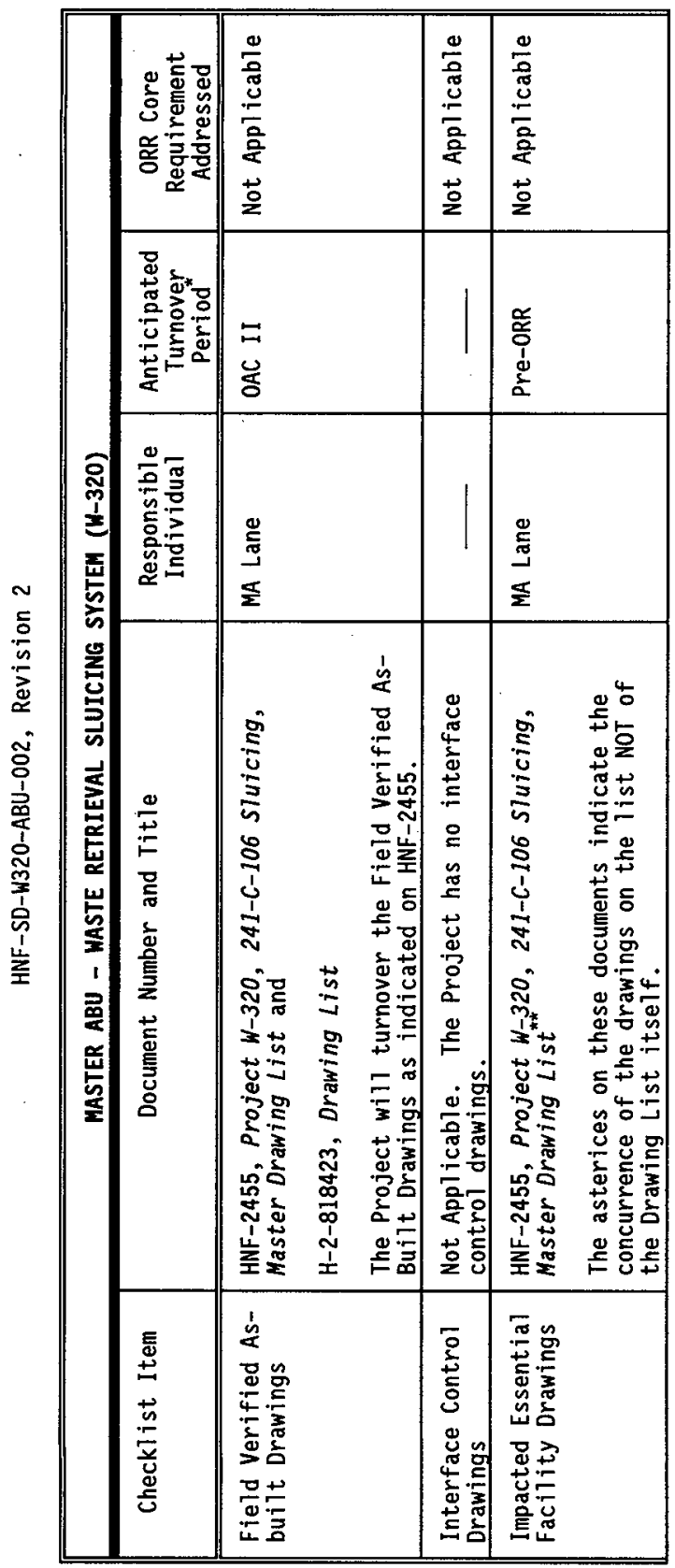




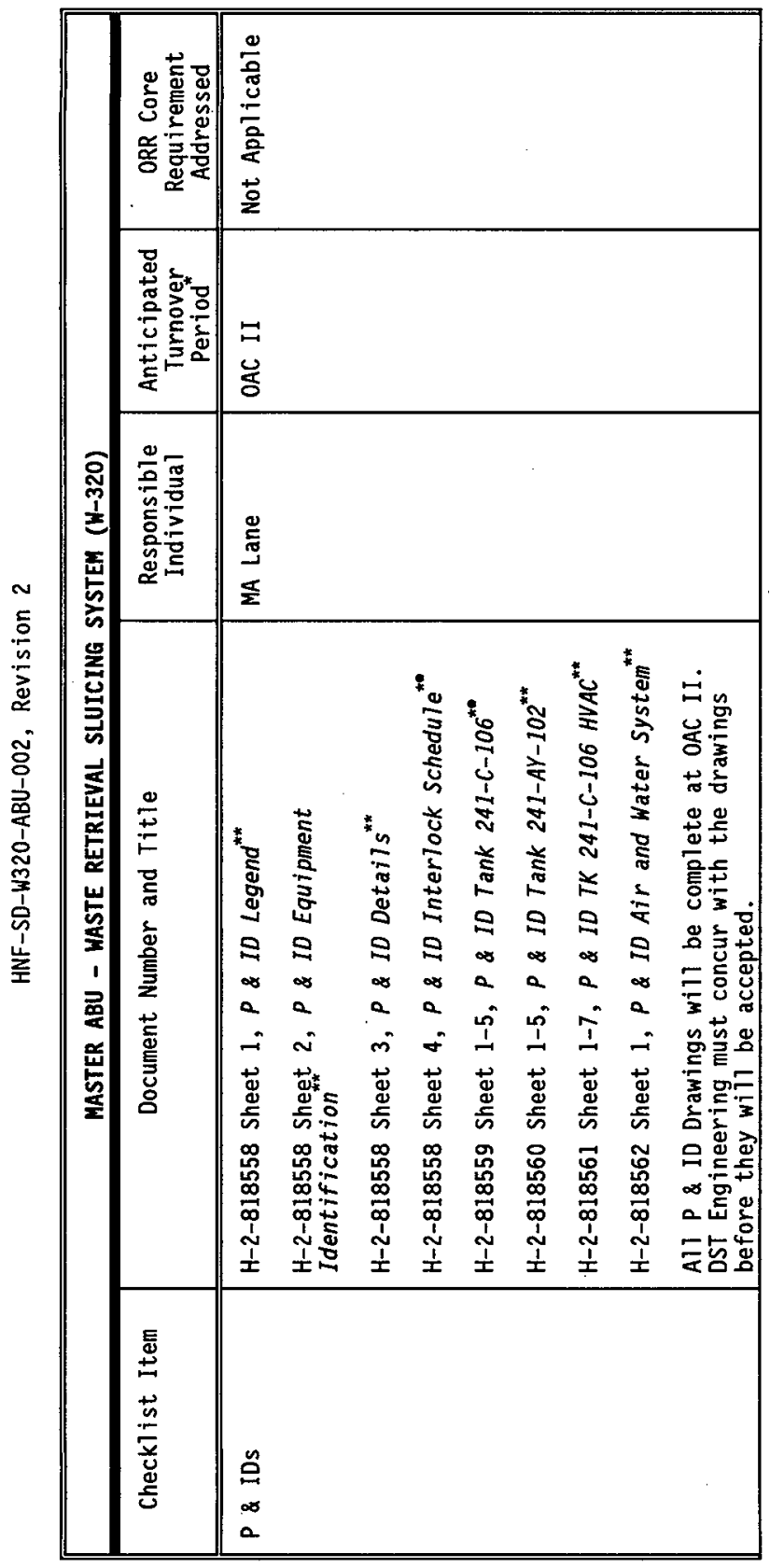




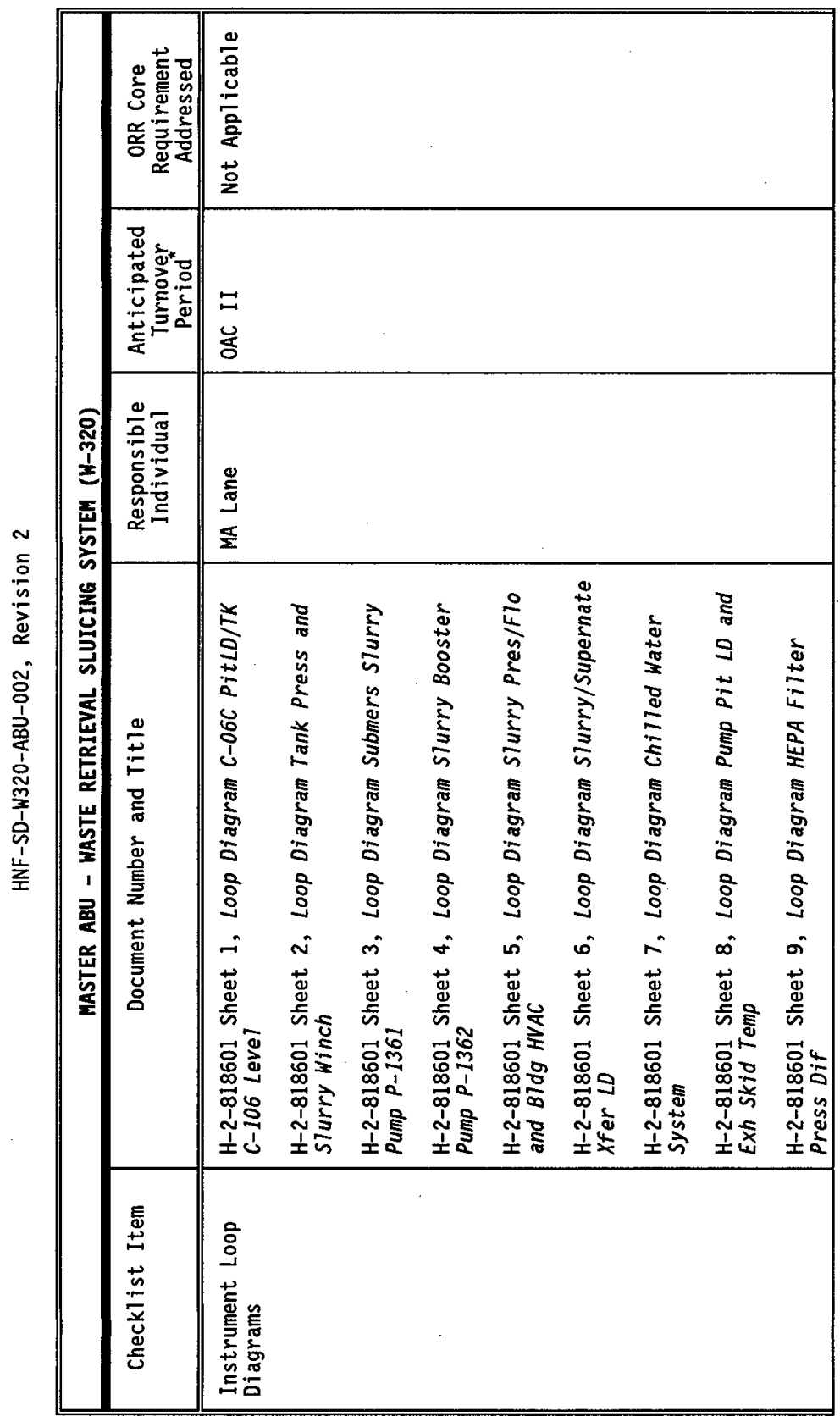




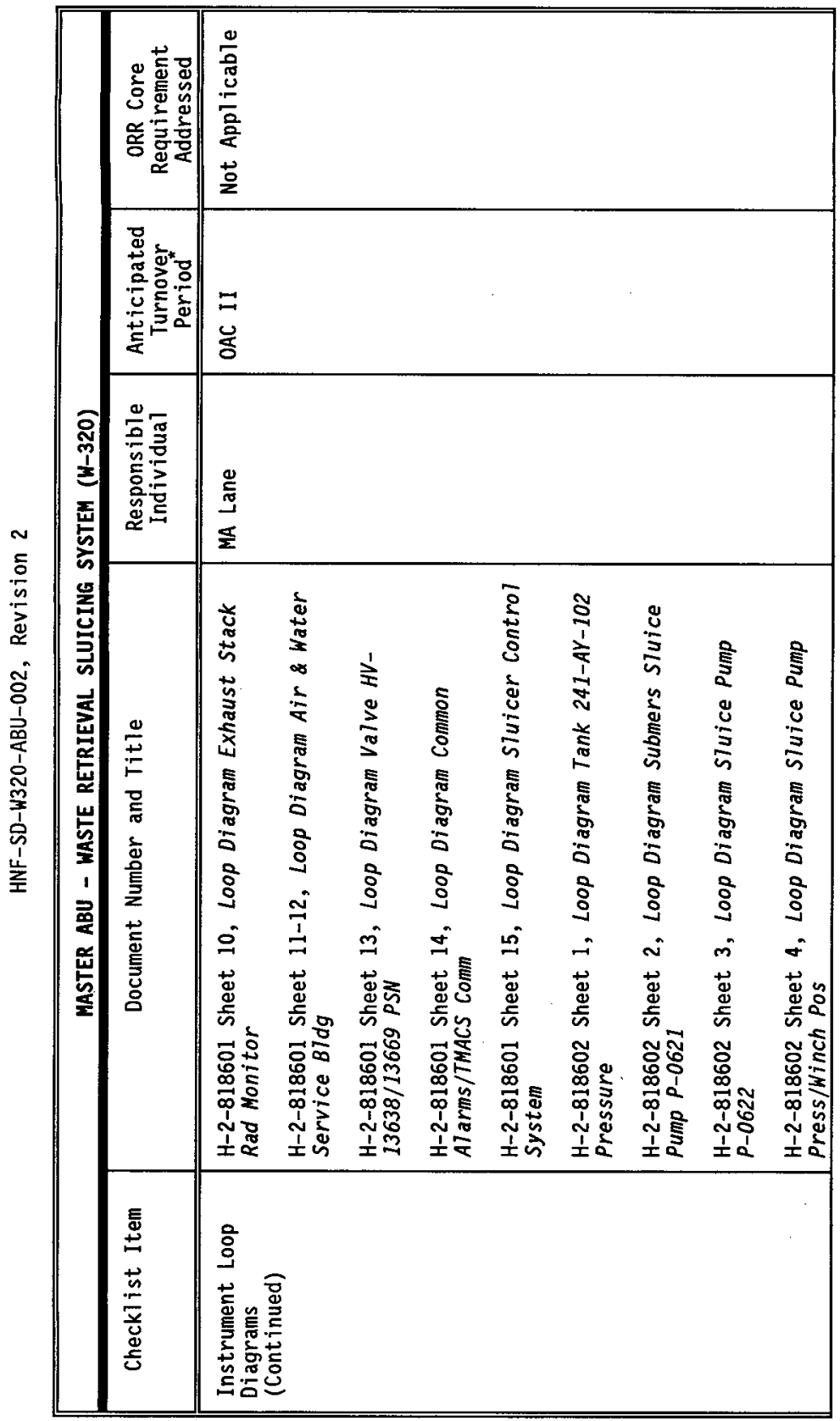




\begin{tabular}{|c|c|c|c|c|c|c|}
\hline \multirow{5}{*}{ 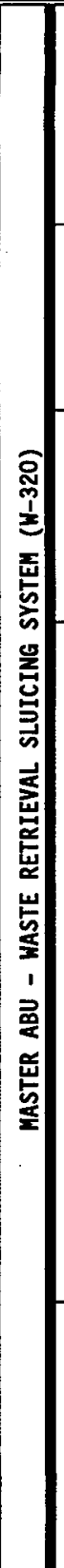 } & 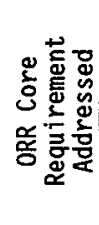 & 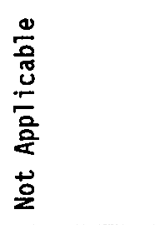 & & & $\begin{array}{l}\frac{0}{0} \\
\frac{0}{0} \\
\frac{0}{0} \\
\frac{a}{2} \\
\overrightarrow{0} \\
2\end{array}$ & 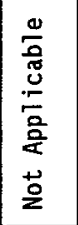 \\
\hline & 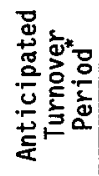 & $\begin{array}{l}\Xi \\
\check{\delta}\end{array}$ & & & & \\
\hline & 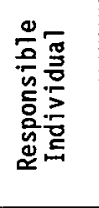 & 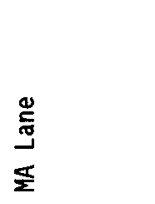 & & & & \\
\hline & 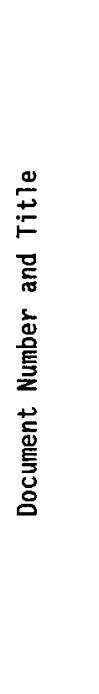 & 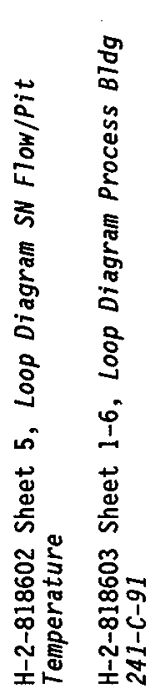 & 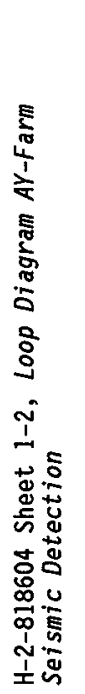 & 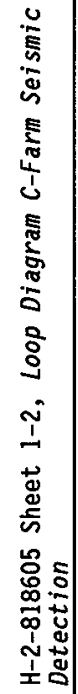 & 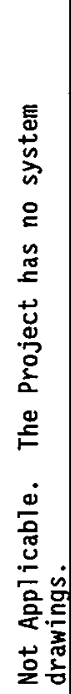 & 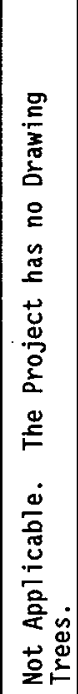 \\
\hline & 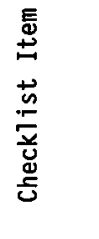 & 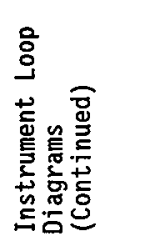 & & & 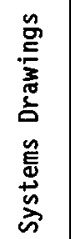 & 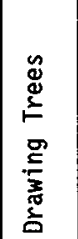 \\
\hline
\end{tabular}




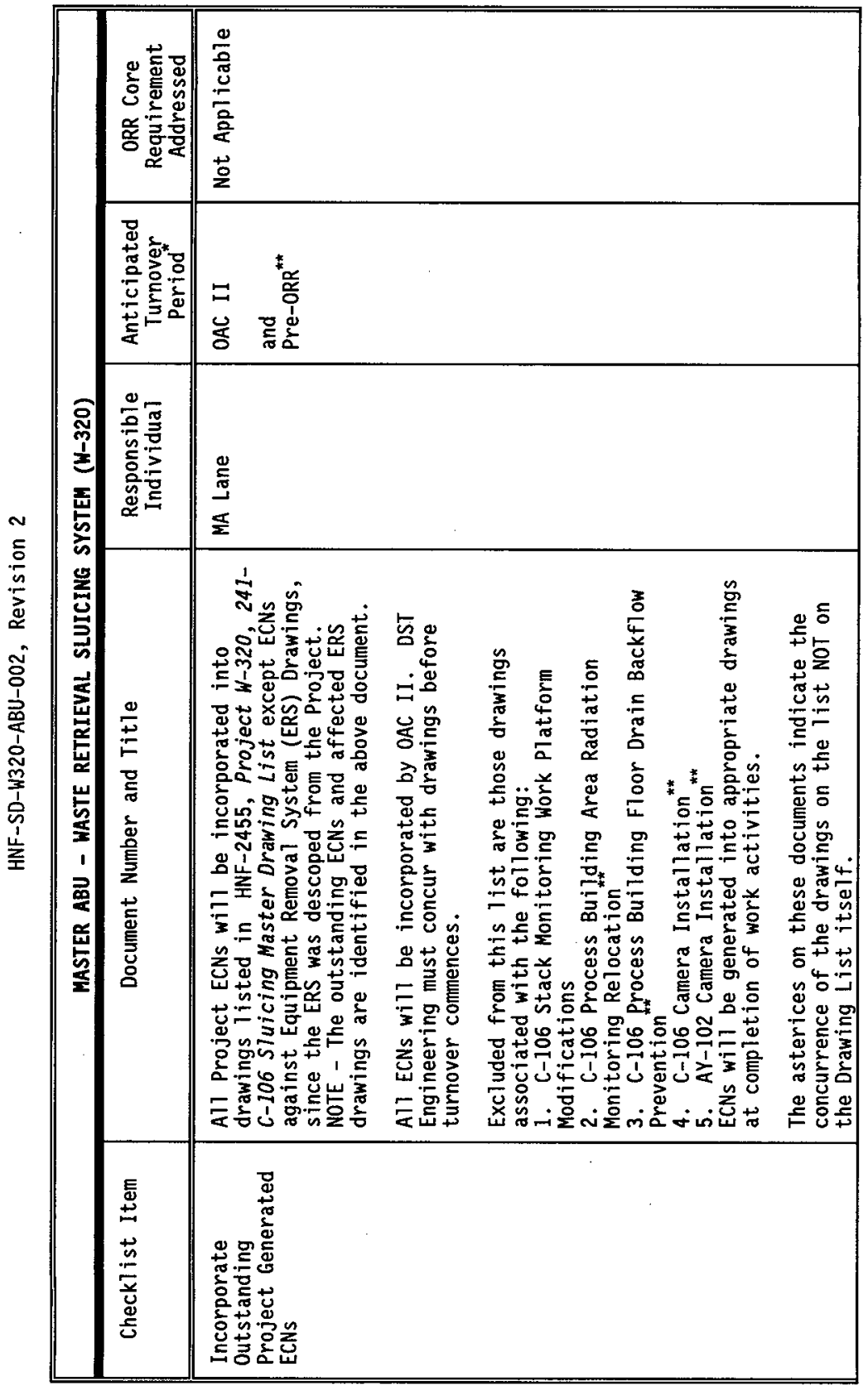




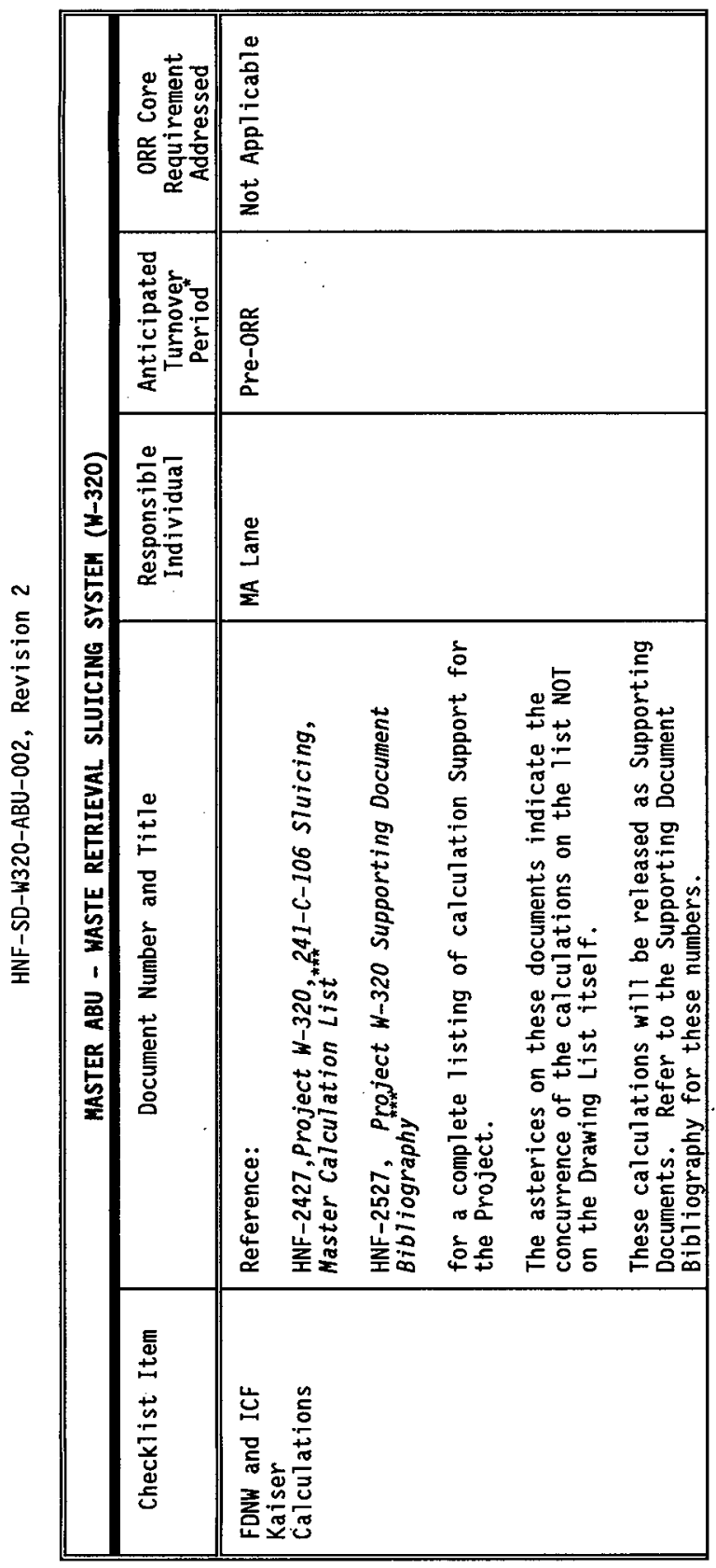




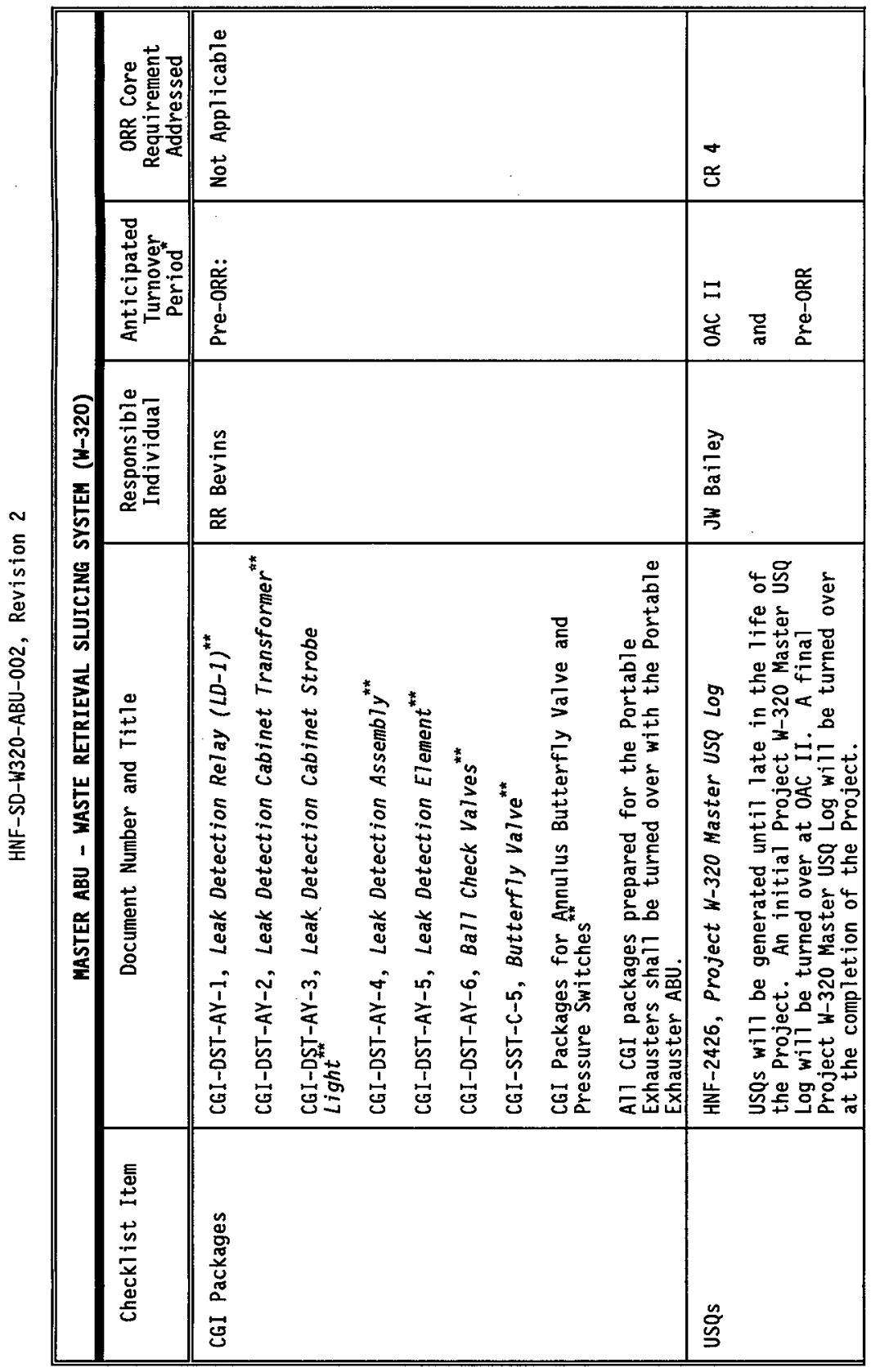

$\infty$
1
1 


\begin{tabular}{|c|c|c|c|c|c|c|c|}
\hline & 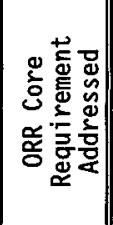 & 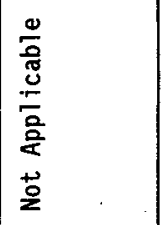 & $\begin{array}{l}\frac{0}{0} \\
\frac{0}{5} \\
0 \\
\frac{0}{2} \\
\frac{0}{2} \\
\stackrel{0}{2}\end{array}$ & 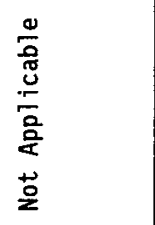 & 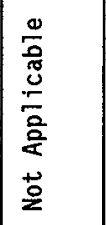 & 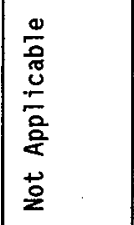 & 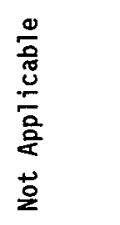 \\
\hline & 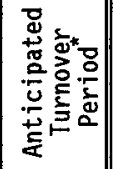 & 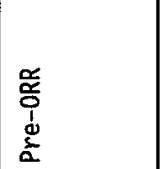 & 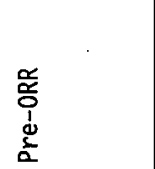 & 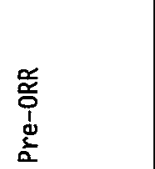 & 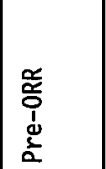 & $\begin{array}{l}\Xi \\
\ddot{y}\end{array}$ & 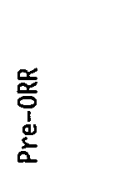 \\
\hline 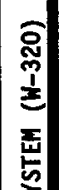 & 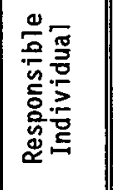 & 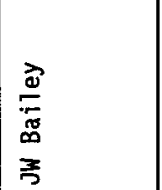 & $\begin{array}{l}\frac{\vec{\varpi}}{\pi} \\
\stackrel{\infty}{\infty} \\
\bar{j}\end{array}$ & 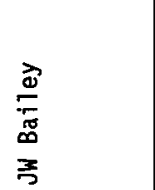 & 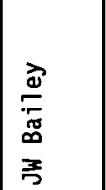 & 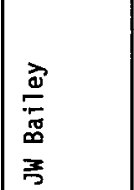 & 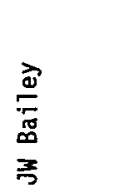 \\
\hline 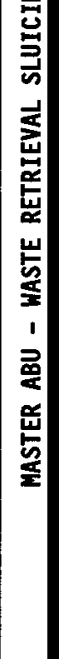 & 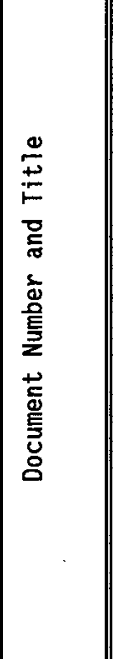 & 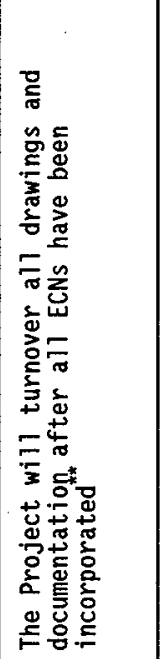 & 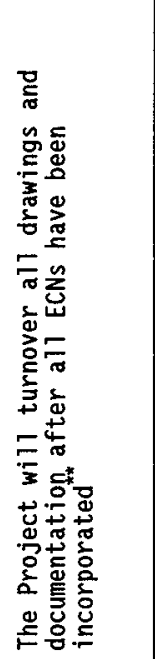 & 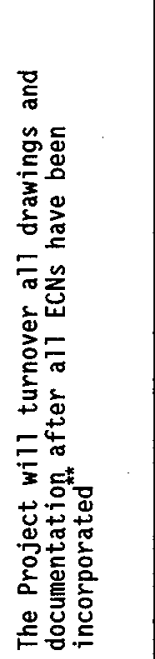 & 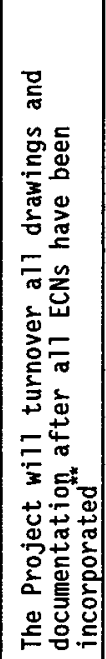 & 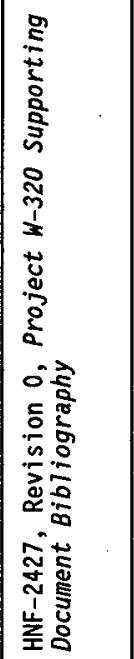 & 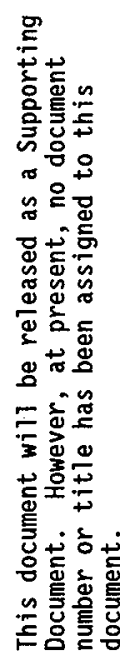 \\
\hline & 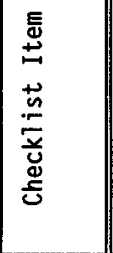 & 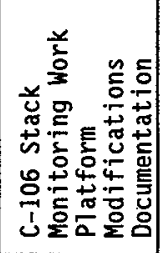 & 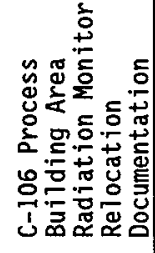 & 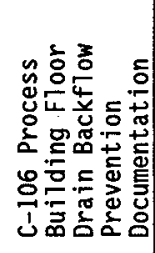 & 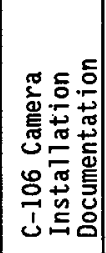 & 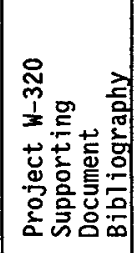 & 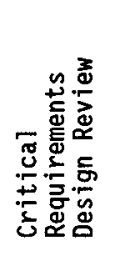 \\
\hline
\end{tabular}




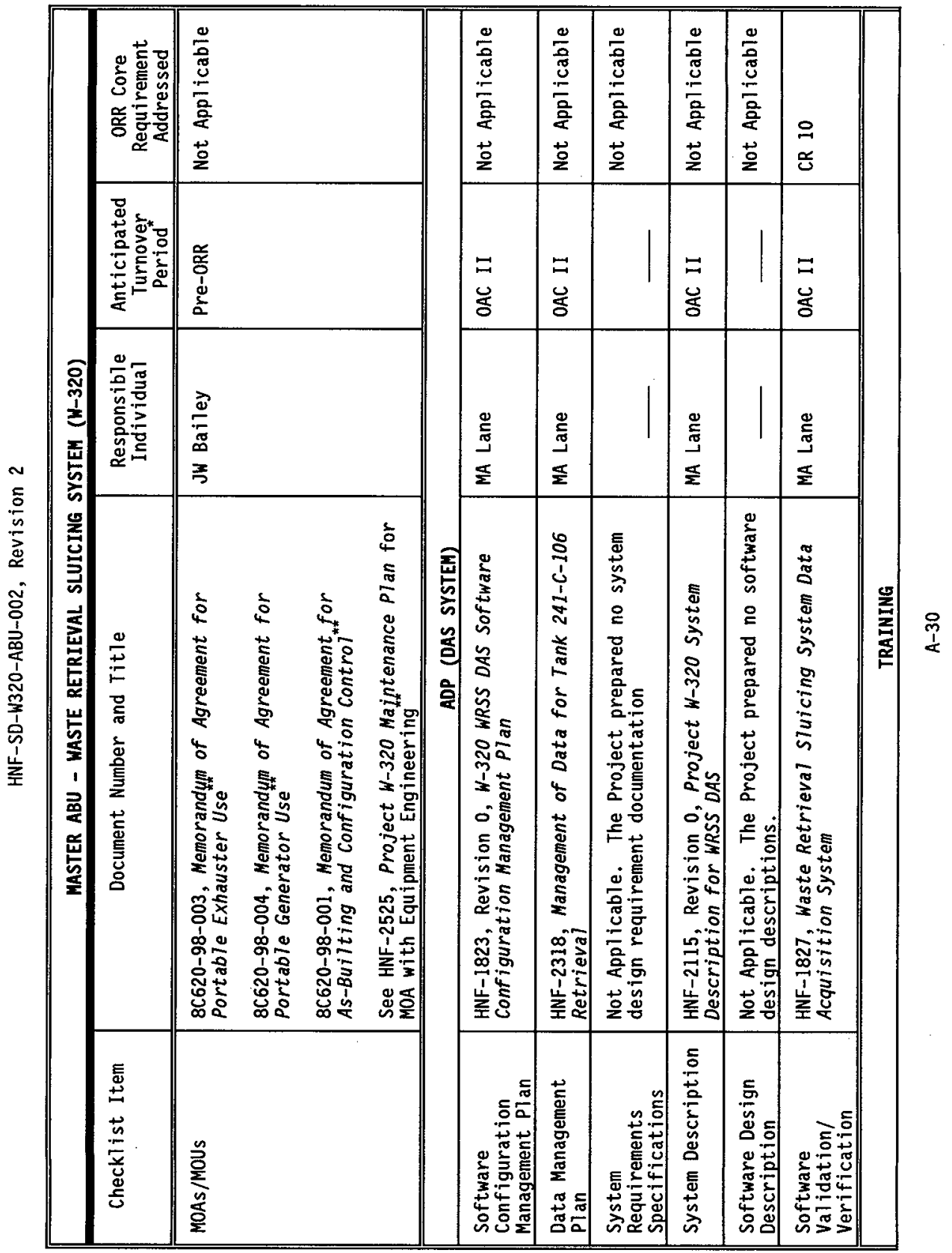




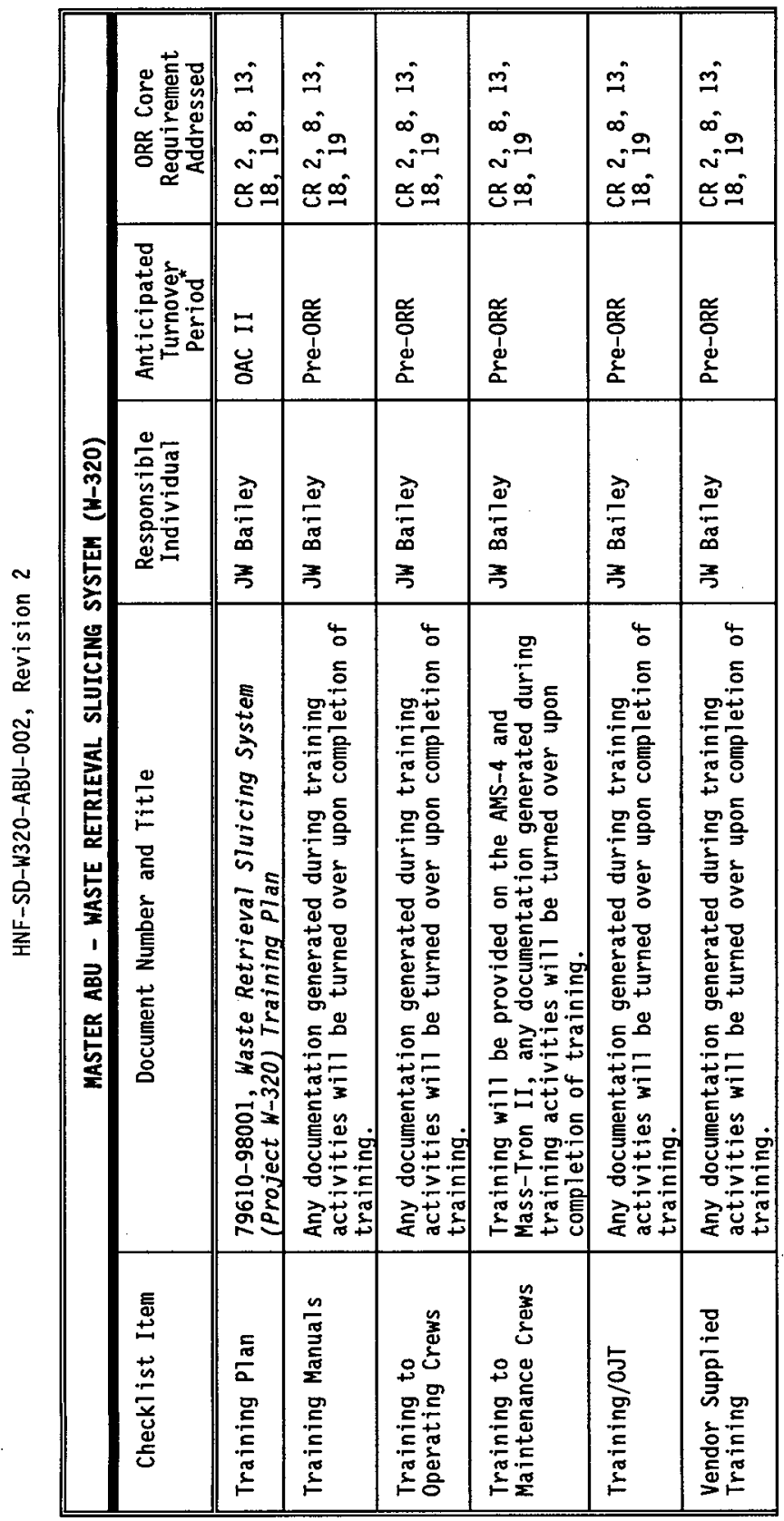




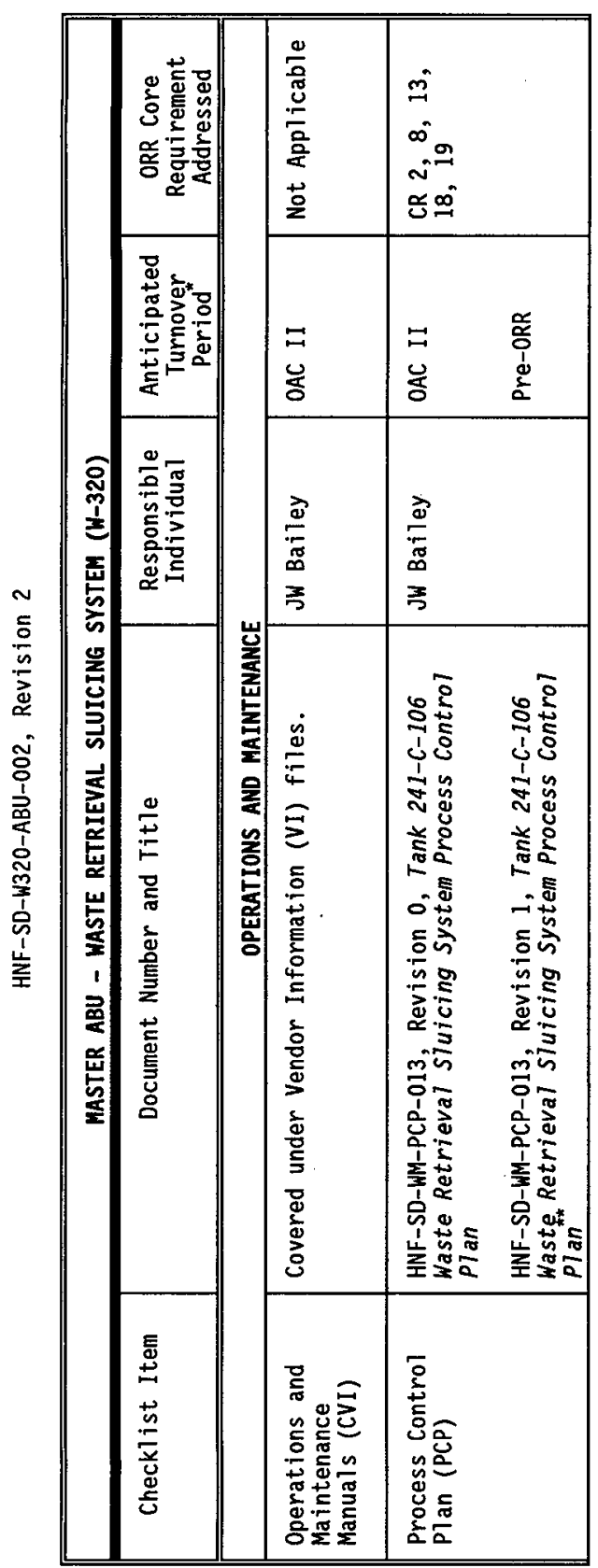




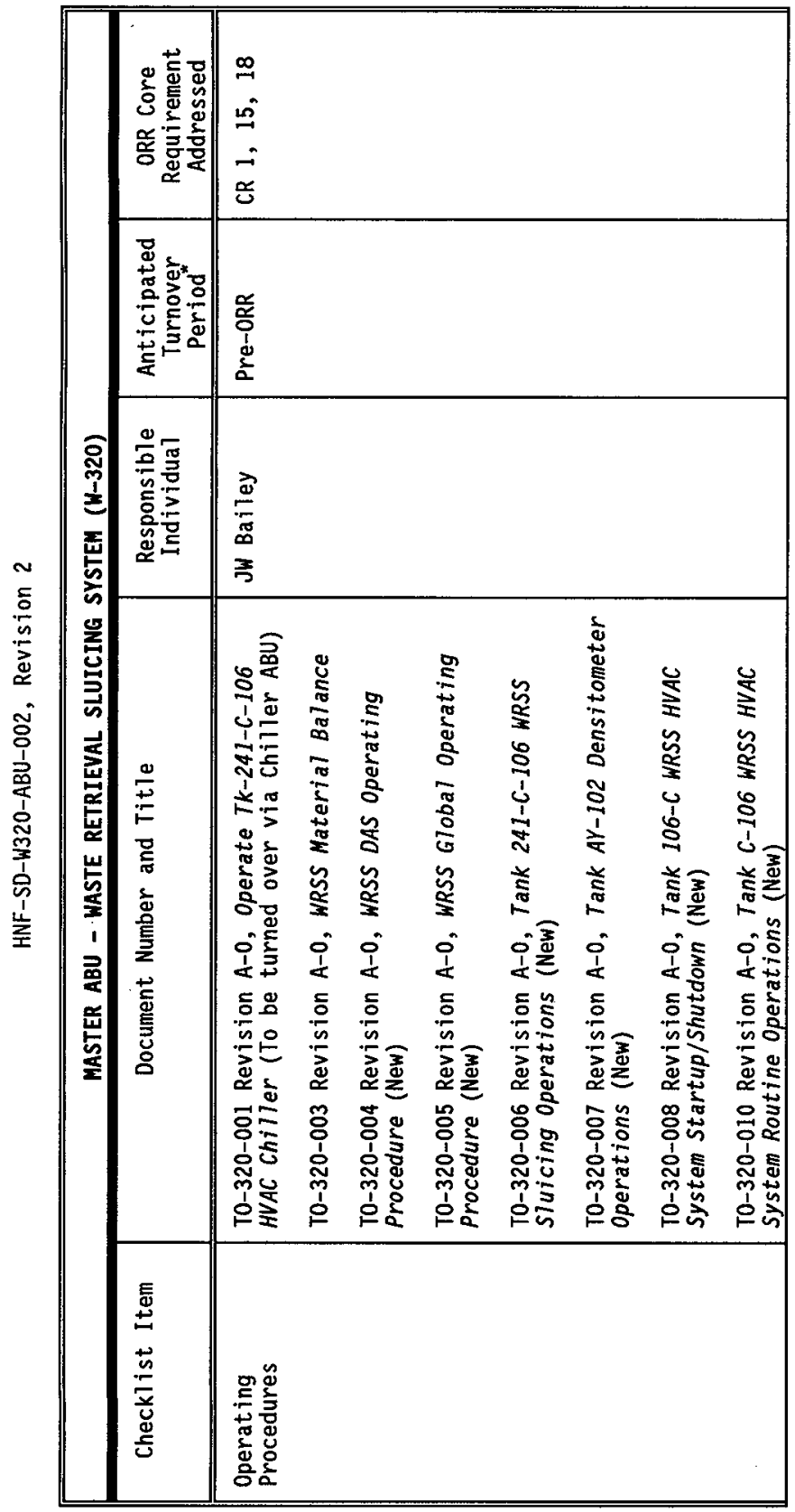




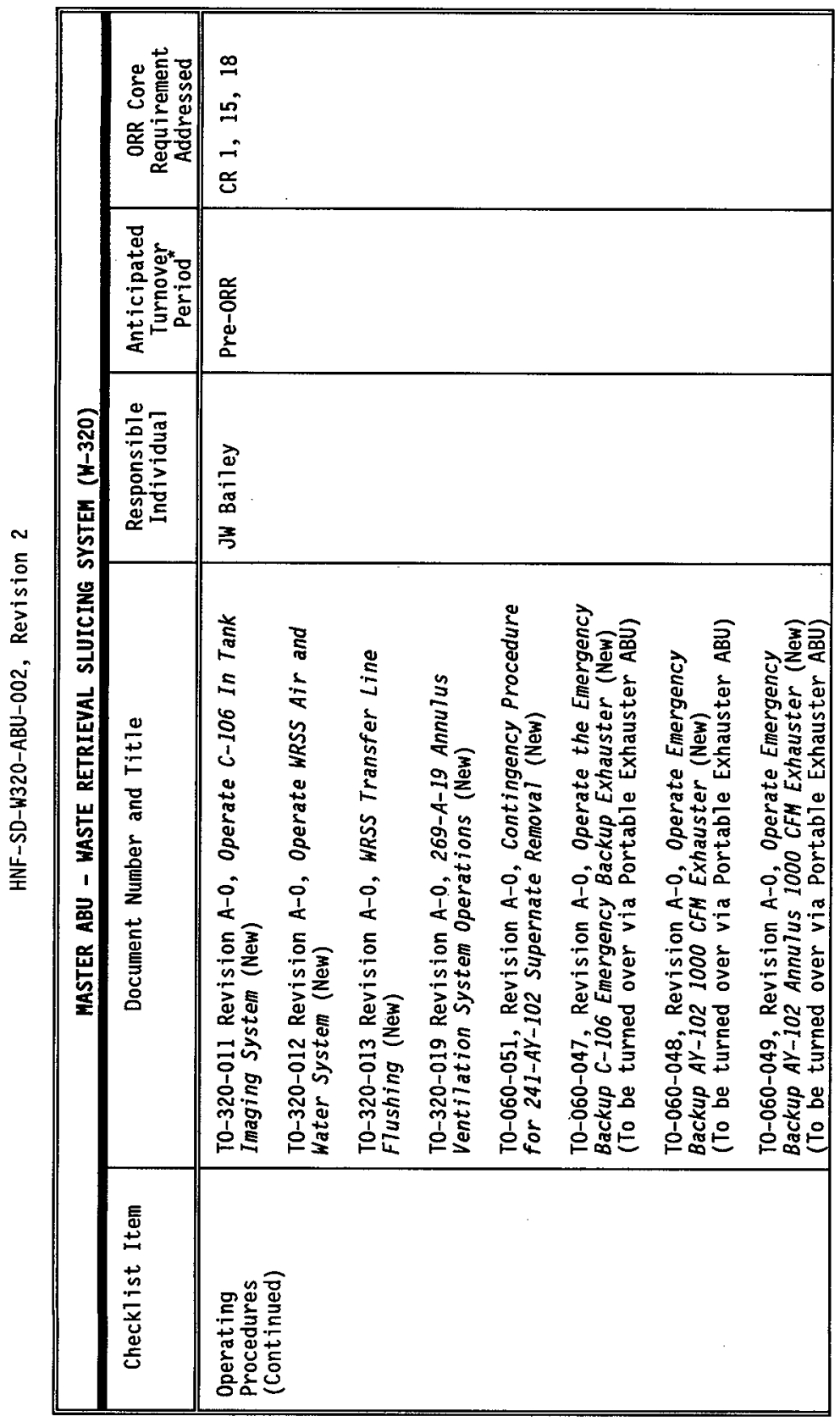




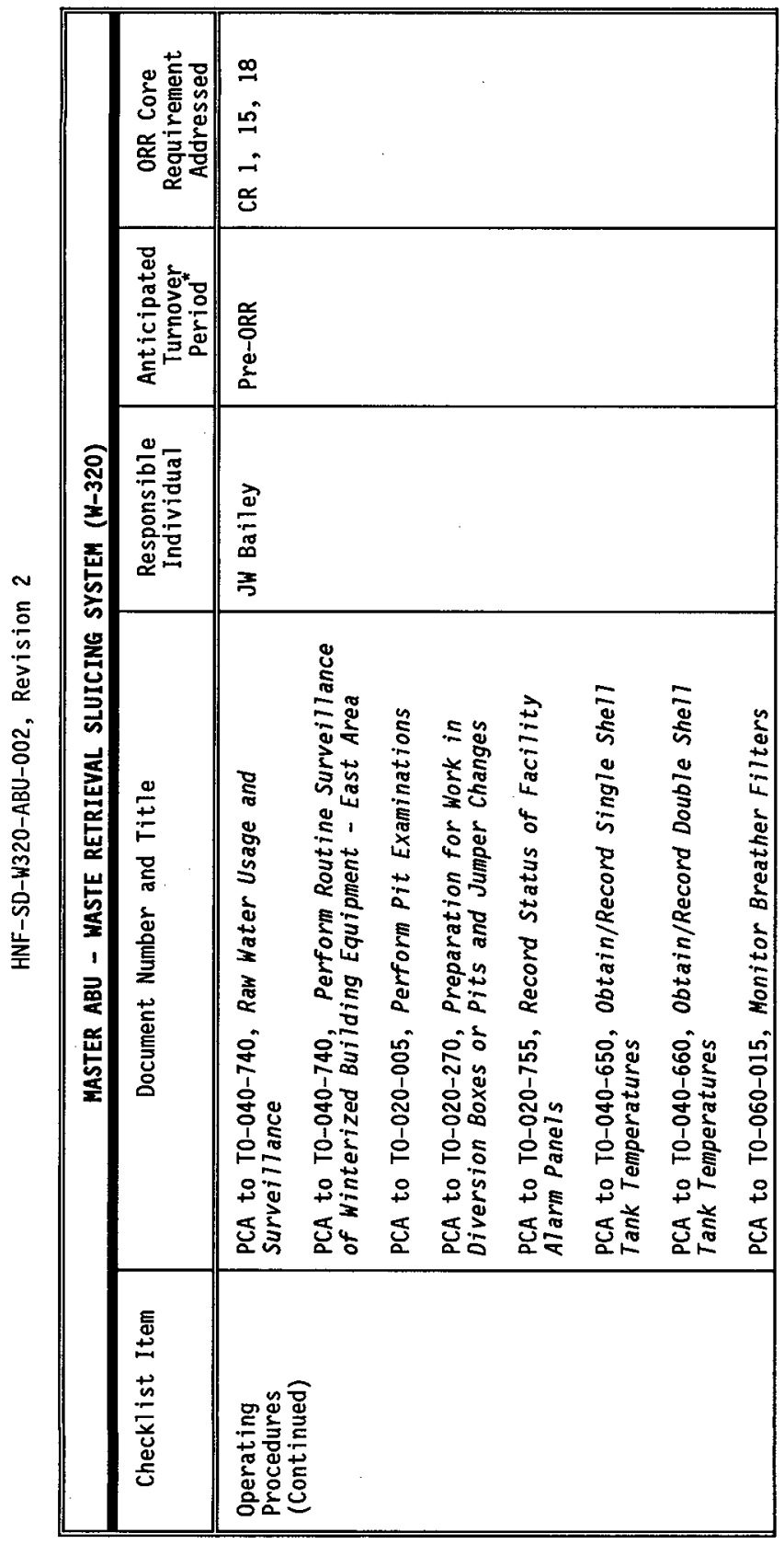




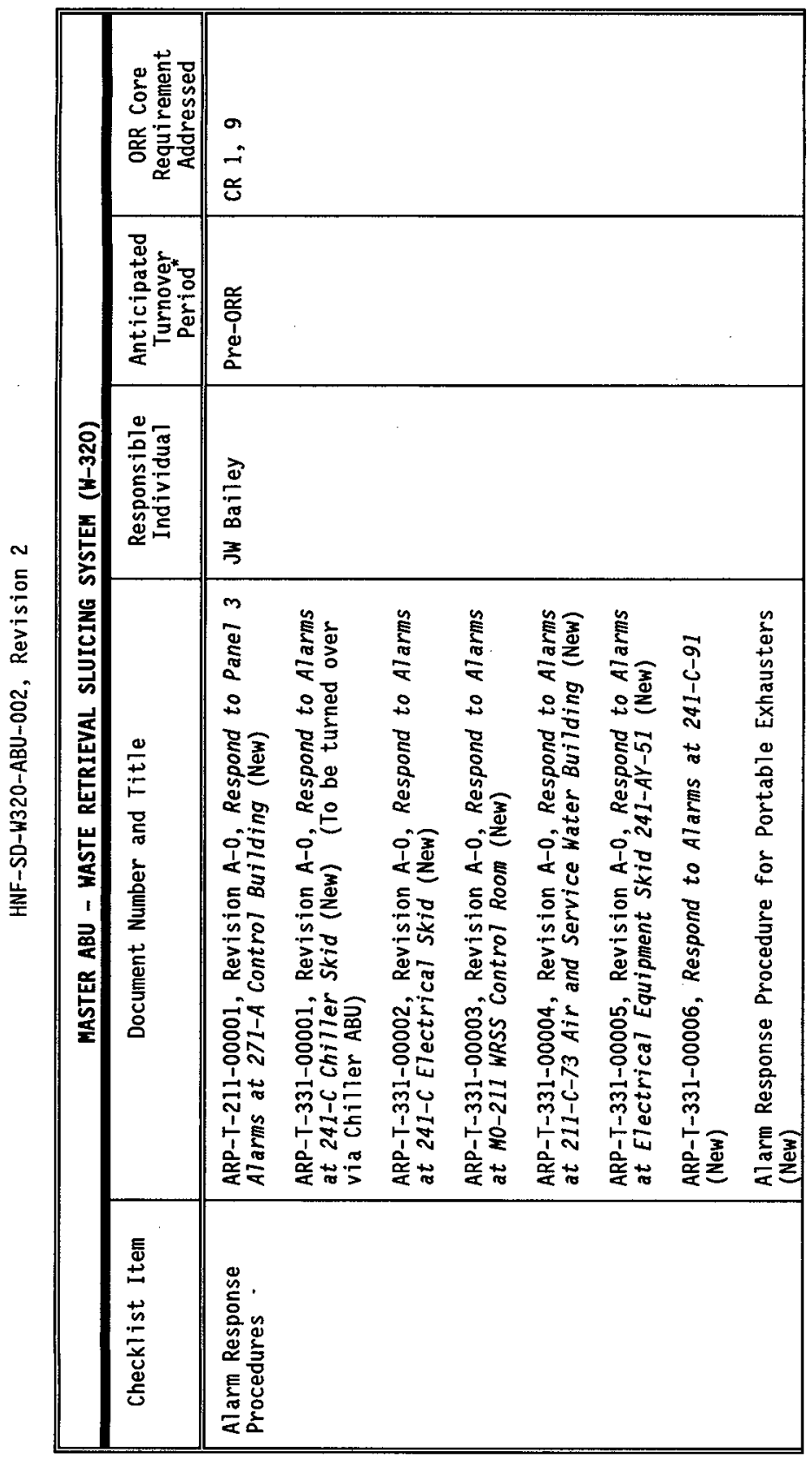




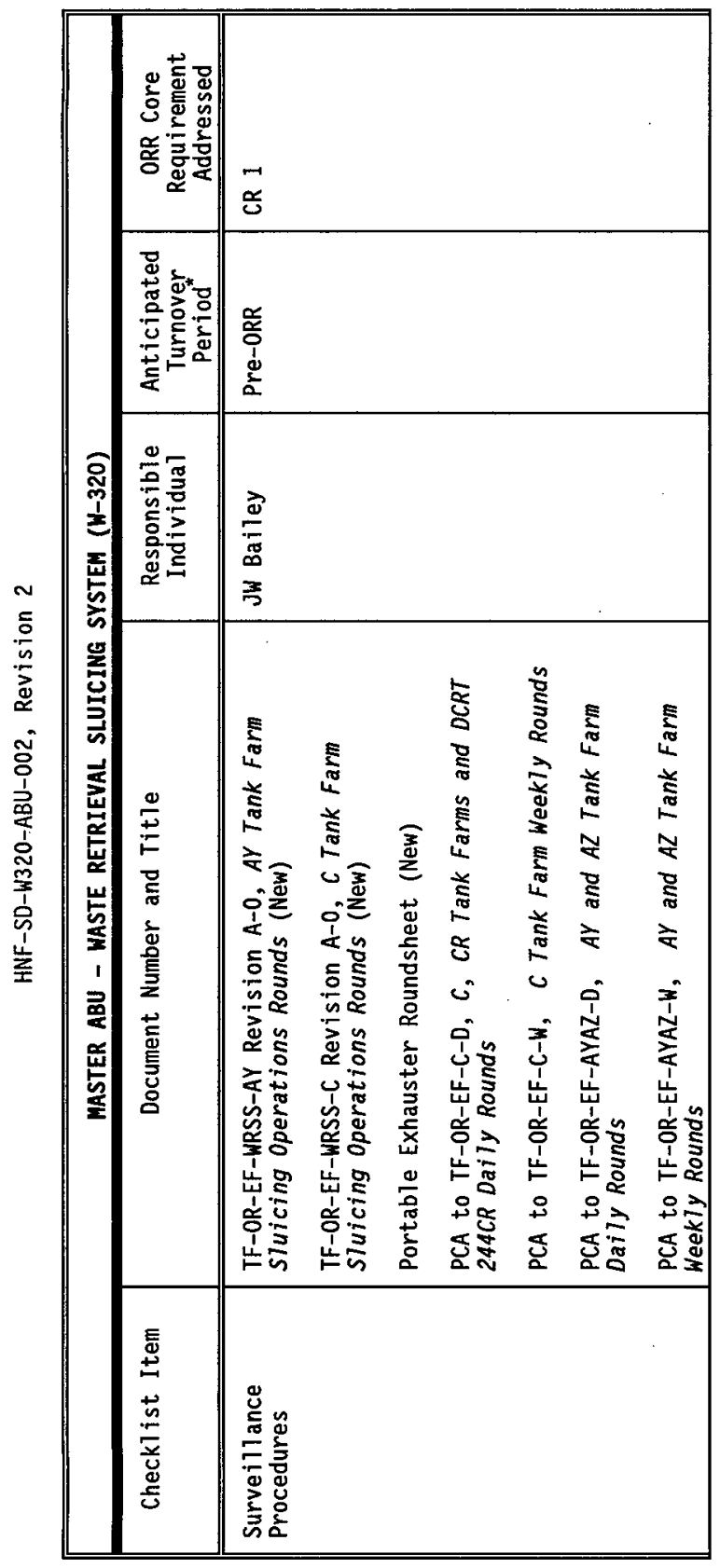

$\frac{1}{2}$ 


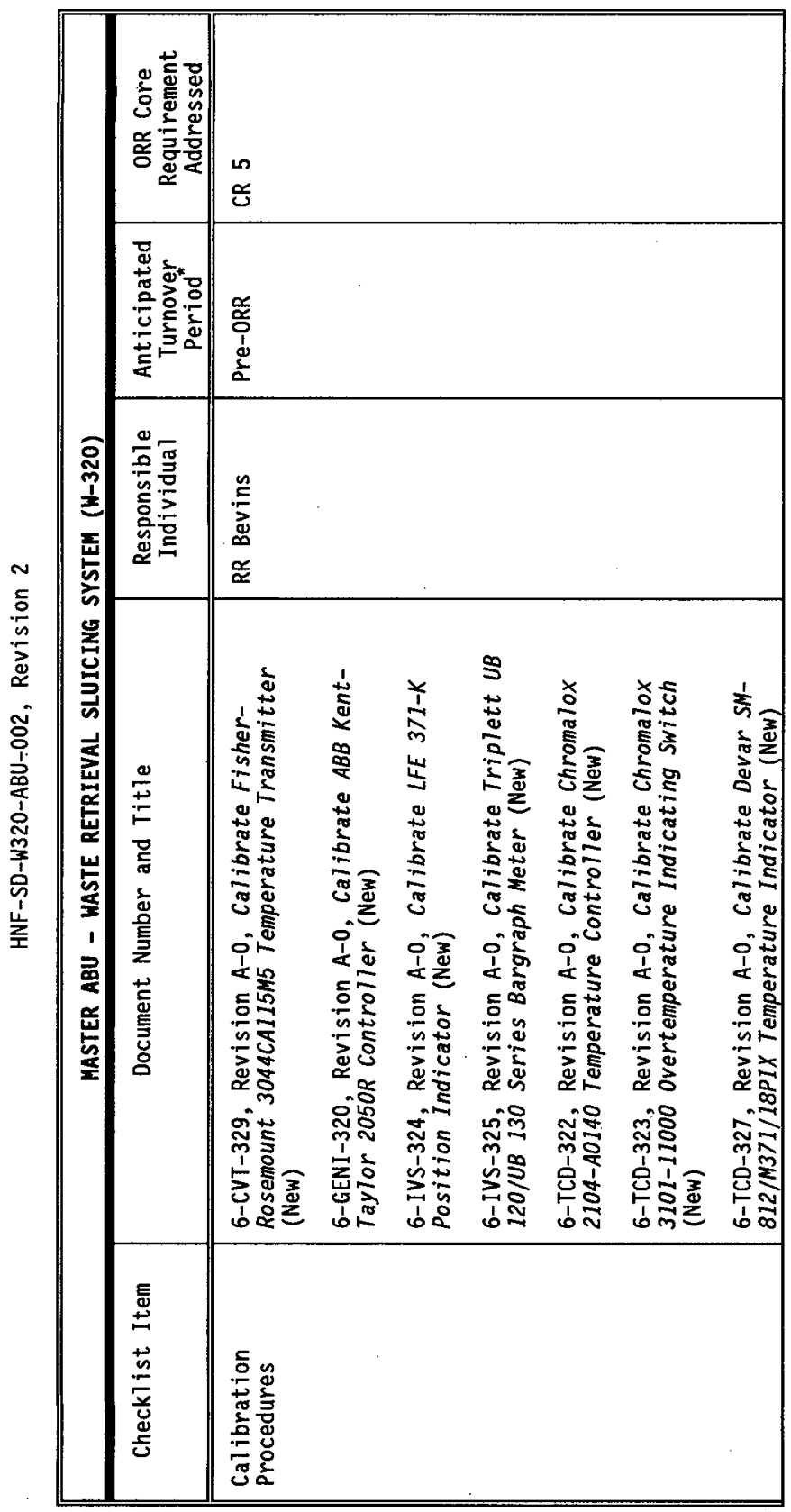




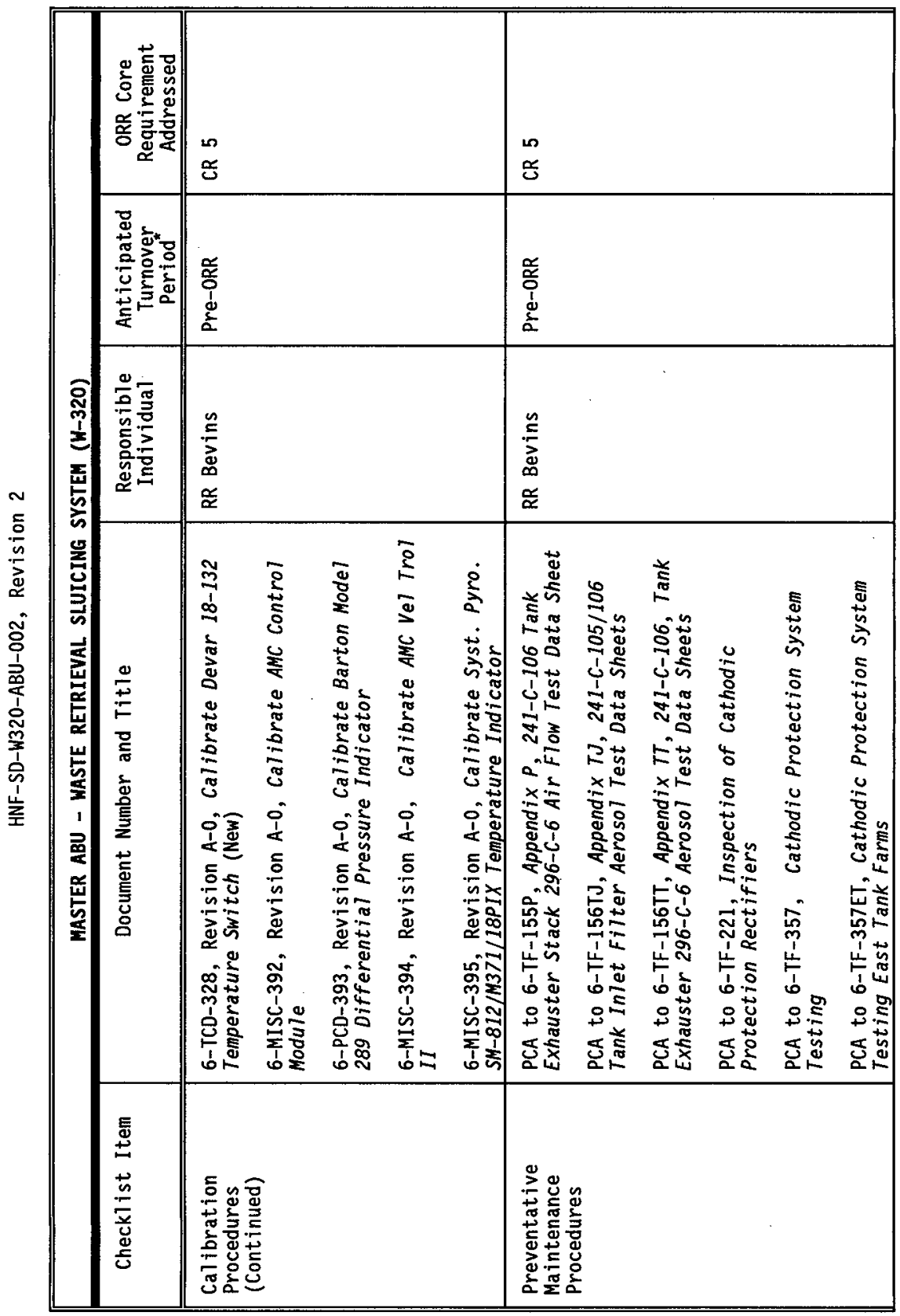




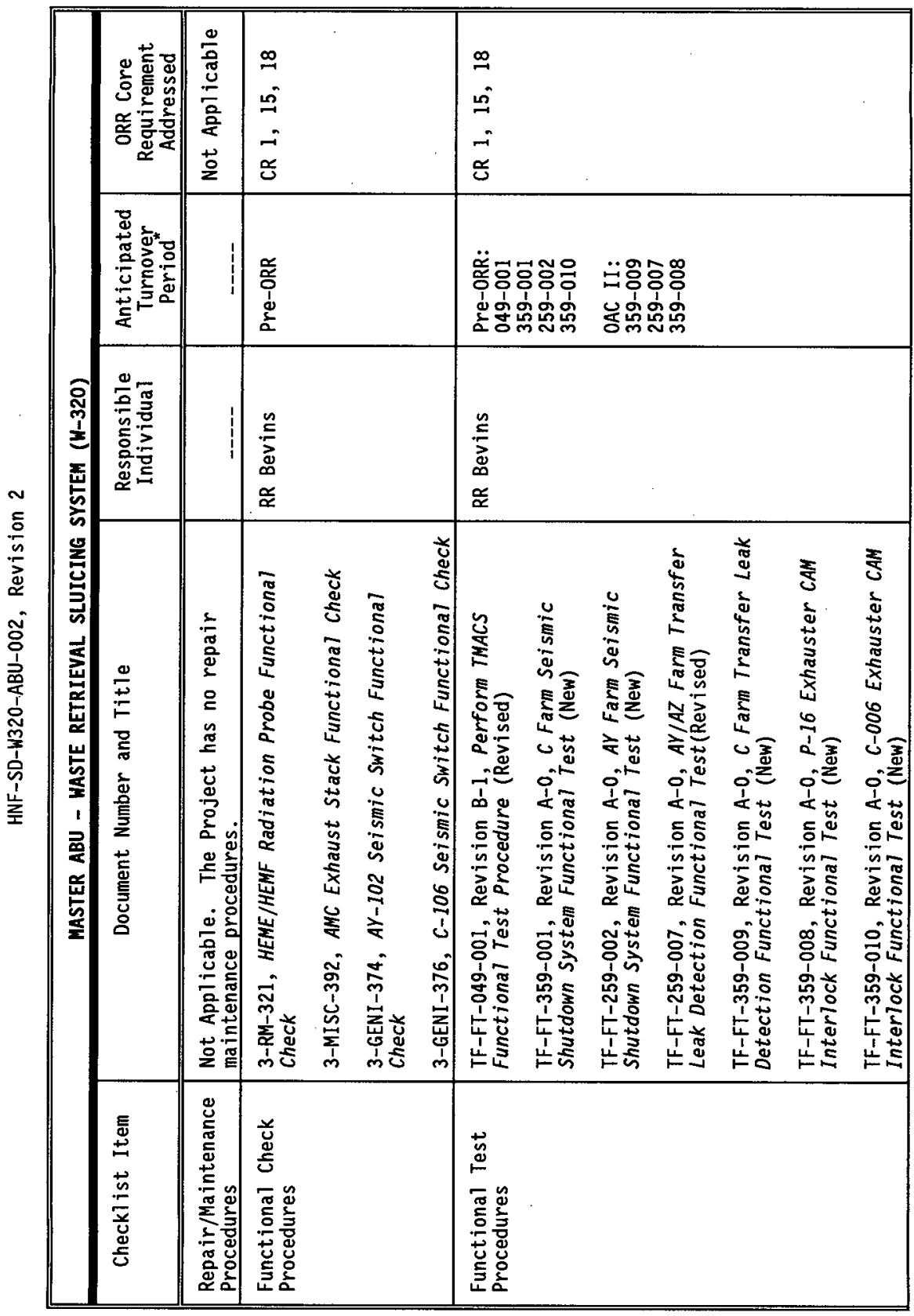




\begin{tabular}{|c|c|c|c|c|c|c|c|}
\hline \multirow{5}{*}{ 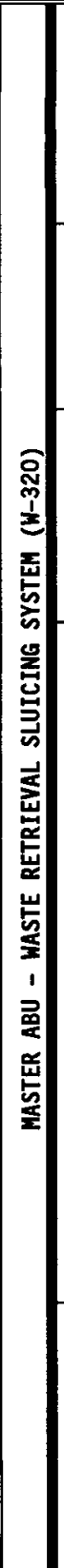 } & 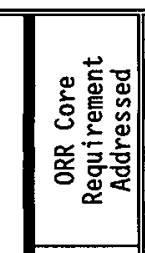 & & \begin{tabular}{|l|}
$\mathbf{2}$ \\
$\simeq$ \\
\end{tabular} & $\begin{array}{l}\text { م } \\
\text { 뚱 }\end{array}$ & & $\begin{array}{l}\frac{0}{0} \\
\frac{0}{0} \\
\frac{0}{2} \\
\frac{0}{2} \\
\overrightarrow{0} \\
\stackrel{0}{2}\end{array}$ & $\begin{array}{l}\frac{0}{0} \\
\frac{0}{0} \\
\frac{0}{0} \\
\frac{0}{2} \\
\stackrel{0}{0} \\
\frac{0}{2}\end{array}$ \\
\hline & 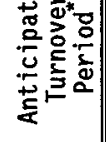 & 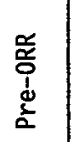 & $=$ & 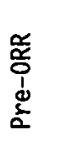 & & $\begin{array}{l}\Xi \\
\stackrel{\Xi}{\delta}\end{array}$ & 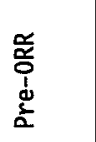 \\
\hline & 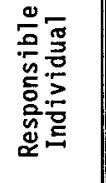 & 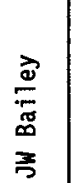 & 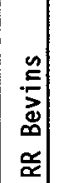 & 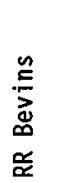 & & 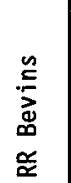 & 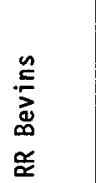 \\
\hline & 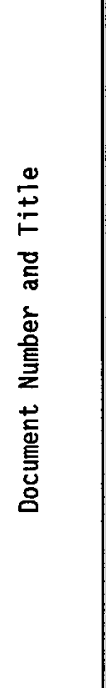 & 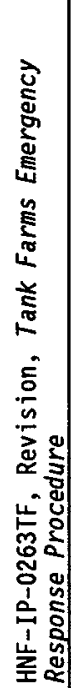 & 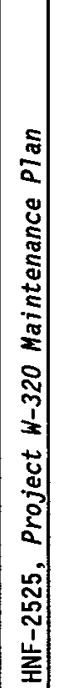 & 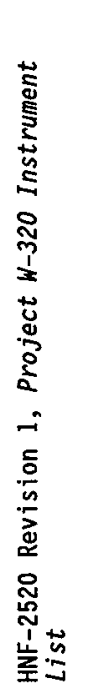 & 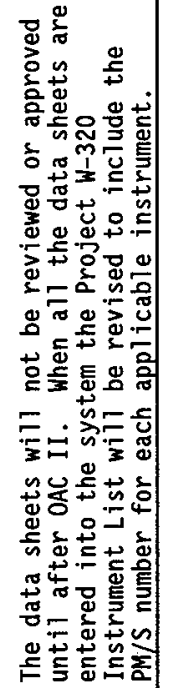 & 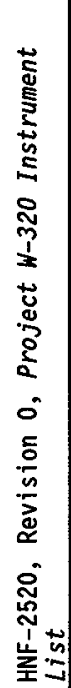 & 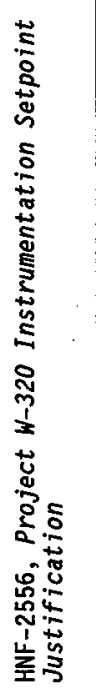 \\
\hline & 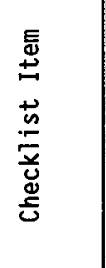 & 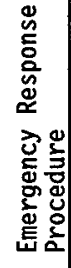 & 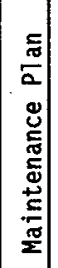 & 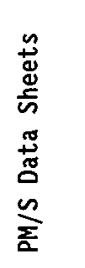 & & 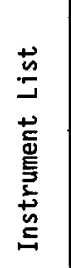 & 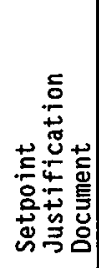 \\
\hline
\end{tabular}




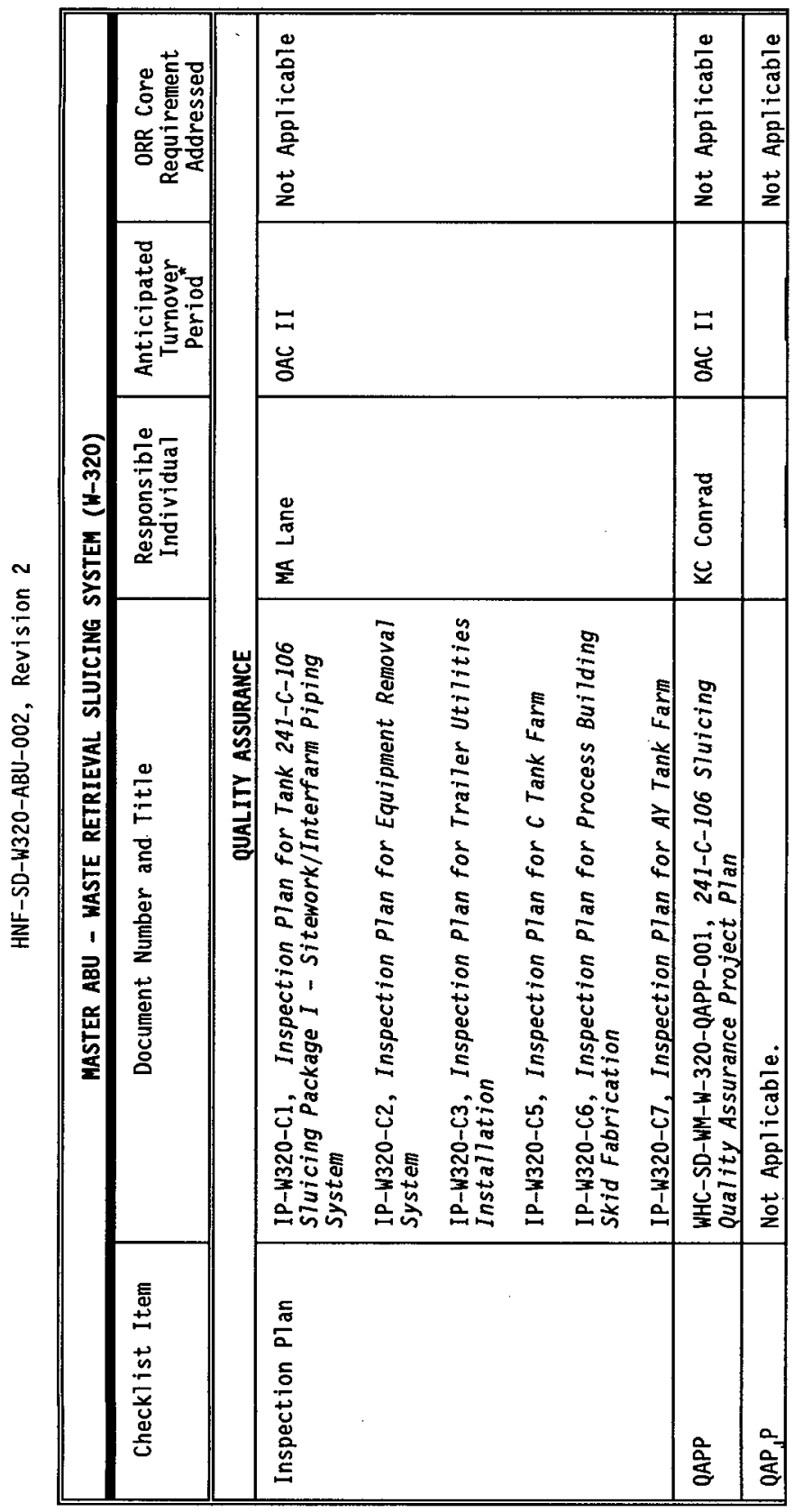




\begin{tabular}{|c|c|c|c|c|c|c|c|c|c|c|}
\hline \multirow{5}{*}{ 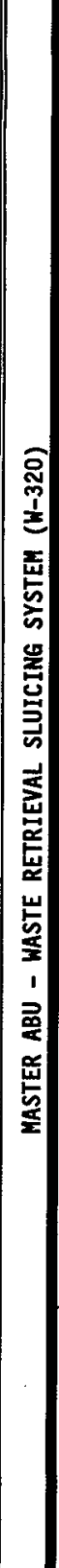 } & 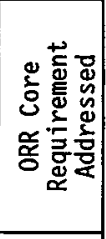 & & 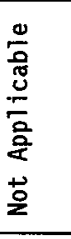 & & & & 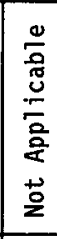 & $\begin{array}{l}\frac{0}{0} \\
\frac{0}{0} \\
\frac{0}{0} \\
\frac{0}{2} \\
\frac{2}{2} \\
0 \\
\frac{0}{2}\end{array}$ & $\begin{array}{l}n \\
\tilde{} \\
\end{array}$ & \begin{tabular}{l}
10 \\
\hdashline \\
\hdashline
\end{tabular} \\
\hline & 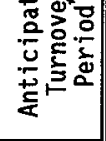 & & $\begin{array}{l}\Xi \\
\text { प̆ }\end{array}$ & 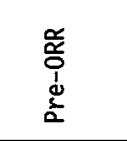 & & & 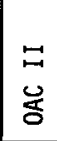 & 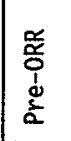 & 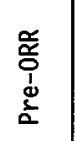 & 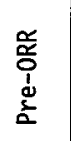 \\
\hline & 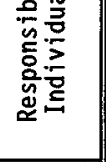 & & $\begin{array}{l}\frac{d}{\pi} \\
\infty \\
\infty \\
\vdots\end{array}$ & & & & 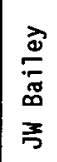 & 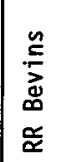 & 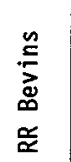 & 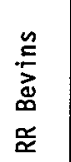 \\
\hline & 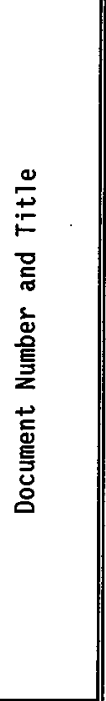 & 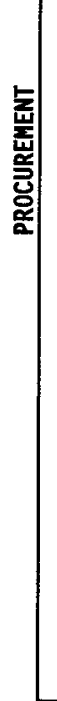 & 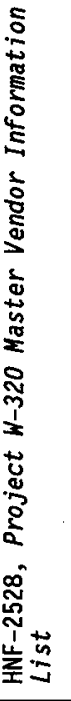 & 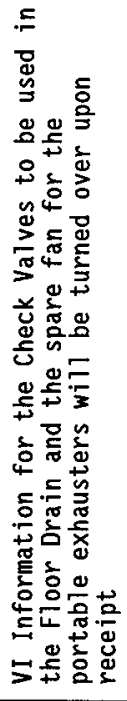 & 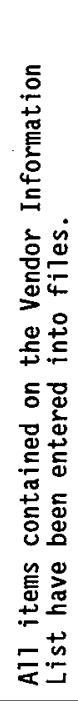 & 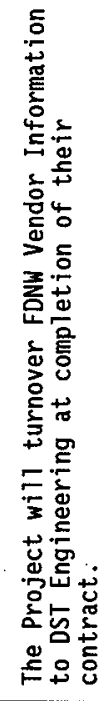 & 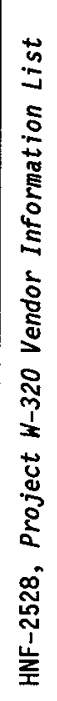 & 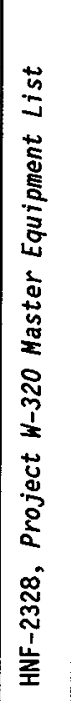 & 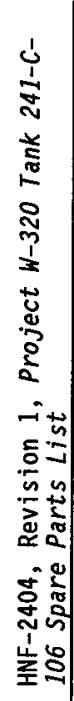 & 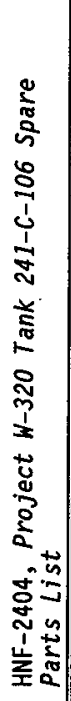 \\
\hline & 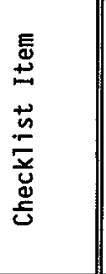 & & 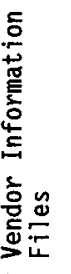 & & & & 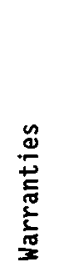 & 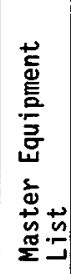 & 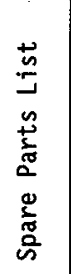 & 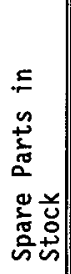 \\
\hline
\end{tabular}


HNF-SD-W320-ABU-002, Revision 2

ATTACHMENT I Correspondence, NHC to FDH, NHC-97571244, Project 93L-EWW-320 "Tank C-106 Sluicing," DOE 6430.1A Criteria Deviation Request 
Numatec Hanford Corporation An SGiv/Cogerns. Inc. Company

August 12, 1997

NHC -9757144

Mr. J. R. Bell, Manager

Industrial Safety \& Fire Protection

Fluor Daniel Hanford

P. 0. Box 1000 GI-15

Richland, Washington 99352

Dear Mr. Be17:

PROJECT 93L-EWW-320, "TANK 24I-C-106 SLUICING, "DOE 6430.1A CRITERIA DEVIATION REQUEST

This letter requests a deviation from criteria contained in U. S. Department of Energy, Richland Operations Office (RL) $6430.1 \mathrm{~A}$ covering automatic sprinkler system requirements for the Process Building covered by this project. This request is supported by a Fire Hazard Analysis (FHA). (copy attached). This analysis has determined that an automatic sprinkler system is not required for this building. The reasoning and technical basis for making this determination have been extracted from the analysis and are summarized below.

This deviation request was originally submitted to Mr. A. W. Kellogg, RL, who has retired. This re-submittal is being made at the request of the RL Project Manager for Project $\mathrm{H}-320$.

Project $W-320$ will provide the facility modifications required to retrieve and transfer high heat wastes from single shell tank 241-C-106 to double shell tank 241-AY-102 using past practice siuicing technology. The transfer of this waste is required to resolve the high heat safety issue for tank 241-C-106.

The project is essentially two sets of pumps, a sluicer, a slurry distributor, two buried pipelines between the two tanks, a new ventilation system for tank 241-C-106, and associated instrumentation, along with operations control stations in both tank farms.

a.) The specific requirement from which a deviation is requested.

A deviation is requested to the criteria for automatic sprinklers as found in DOE 6430.1A, Section 1530-99.0, which requires automatic water sprinkler coverage throughout the Process Building. 
b.) Analysis of hazards including the nature. magnitude, and conseouences.

As discussed in the FHA, a review of the equipment and instrumentation drawings indicates that there are no significant combustible loadings. The only identified ignition source results from electrical failure. Any resulting fire is expected to be limited in size and is not expected to spread due to the very low combustible loading. Automatic sprinkler protection is not expected to prevent or reduce any subsequent loss that may result from an electrical failure, and even if an external fire were to occur and involve the Process Building, the consequences would not exceed $\$ 1,000,000$ or result in adverse health impacts to the onsite or offsite individual beyond DOE required limits. Consequently, automatic water sprinkler protection is not recommended.

c.) The soecific "effects" on the maanitude for an incident that can be attributed to noncompliance.

The Process 8uilding is located in developed areas where fire hydrant protection is provided. Replacement costs associated with a maximum credible fire (MCFL) would be increased to equal that of the maximum possible fire (MPFL). However, the dollar loss would still be within DOE 5480.7A and 6430. IA limits for such a loss in an unsprinklered facility. Additionally, the 200 Area fire station provides a fiveminute response time to the building. This will likely limit the loss to minimal levels.

d.) All necessary and applicable drawings, specifications, etc.. depicting the changes that would result from the deviation.

Oniy the design media for Project $W-320$ would be affected by this deviation.

e.) The reasoning and technical basis for the conclusion that noncompliance is acceptable.

- The possibility of a fire is remote because of the noncombustible building construction and the low fuel loading in the building.

- Radiological or toxicological release are not postulated to occur as a result of a MPFL event.

- The replacement cost of a building in the event of a fire is less than $\$ 1,000,000$. See the attached preliminary FHA HHC-SD-W320FHA-001, Rev. 0 . Please note that the FHA did not provide deposition and cleanup cost analysis. Property loss figures were exaggerated to account for the fact that the facility is located in a radiation zone. This is a reasonable method and is likely to be conservative for calculating the consequences based on a graded approach and negligible contamination in and around the building. 
- Portable fire extinguishers (type $A B C$ dry chemical) will be provided.

- The Process Building is located in a developed area which has adequate fire hydrant protection.

- The 200 Area fire station provides a five-minute response time to any of the new buildings.

- There will be no permanent occupancy of the new buildings. Maintenance and operations personnel will periodically occupy the buildings on a temporary basis to perform assigned duties.

- The maximum credible fire loss is the same as the maximum possible fire loss, which is below the level that would require automatic fire protection.

\section{f.) The term for which the deviation is required.}

The deviation is permanent for the 1 ife of Project $W-320$.

This request has been discussed with Messrs. W. Abdul and C. P. Christenson. If you have any questions regarding this subject, please contact Messrs. J. W. Bailey on 372-0045 or N. F. Barilo on 372-3191.

Very truly yours,

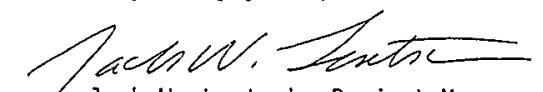

Jack W. Lentsch, Project Manager

Numatec Hanford Corporation

cjh

Attachment 
HNF-SD-W320-ABU-002, Revision 2

ATTACHMENT 2 Correspondence, FDH to DOE, FDH-9757289, Contract DE-AC06-96RL13200, Secti H-14, Laws, Regulations, and DOE Directives - Deviation Request for DOE 6430.1A, "Gener Design Criteria" 


\section{FLUOR DAMIEI}

Fluor Daniel Hanford. Inc.

P.O. $80 \times 1000$

Richland, WA 99352

August 20, 1997

FDH-9757289

Mr. J. E. Kinzer, Assistant Manager

Tank Waste Remediation

U.S. Department of Energy

Richland Operations office

Post Office Box $550 \quad 57-50$

Richland, Washington 99392

Dear Mr. Kinzer:

CONTRACT DE-AC06-96RL13200, SECTION H-14, LAWS, REGULATIONS, AND DOE DIRECTIVES DEVIATION REQUEST FOR DOE 6430.1A, "GENERAL DESIGN CRITERIA"

Reference: Letter, S. R. Morgan, WHC, to A. H. Kellogg, RL, "Project 93L-EWW320, Tank 241-C-105 Sluicing, DOE 6430.1A Criteria Deviation Request," \#9556514 dated January 9, 1996.

The subject contract section requires compliance with DOE 6430.1A, "Generai Design Criteria." In turn, DOE 6430.1A, Section 1530-99.0, requires the installation of automaic sprinkler protection.

Fluor Daniel Hanford, Inc., (FDF) is requesting a permanent deviation from this requirement for Project 93L-EWW-320, "Tank 241-C-106 Sluicing." This request was originally submitted (by the referenced letter) to Mr. A. W. Kellogg, RL, who has retired, and was reviewed by RL's Quality, Safety, and Health Programs Division (QSH). However, a response was never received and the deviation is being resubmitted as requested by the RL Project Manager (Mr. W. AbduT).

The Fire Hazards Analys is (FHA) for Project $93 \mathrm{~L}-E W W-320$ is attached as a supporting document for the deviation request. The FHA is al so being included for review by RL's QSH Division as required by RLID 5480.7, "Fire Protection," Section 6.2.e. 
HNF-SD-W320-ABU-002, Revision 2

ATTACHMENT 3 Correspondence, 97-WSD-255, Contract Number DE-AC-06-96RL13200 - Section H 14, Laws, Regulations and U.S. Department of Energy (DOE), Richland Operations Office ( $R$ Directives - Deviation Request for 6430.1A "General Design Criteria" for Project W-320 Tank C-106 sluicing 
Mr. H. J. Hatch, President

Fivor Danlel Hanford, Inc.

Richland, Washington 99352

Dear Mr. Hatch:

CONTRACT NUMBER DE-AC06-96RL]3200 - SECTJON H-14, LAKS, REGULATIONS, AND U.S. DEPARTMENT OF ENERGY, (DOE), RICHLAND OPERATIONS OFFICE (RL) DIRECTIVES DEVIATION REQUEST FOR 6430.1A, "GENERAL DESIGN CRITERIA" FOR PROJECT W-320, TANK C-106 SLUICING

In reference to the Fluor Daniel Hanford, Inc. letter from $A$. M. Umek to J. Kinzer, RL, "Section H-14, Laws, Regulations, and DOE Directives Deviation Request for DOE 6430.1A, "General Design Criterfa', "FOH-9757289, dated August 20, 1997, RL has reviewed the Fire Hazard Analysis in support of the deviation request. The deviation request has been revtewed in accordance with RLID 5480.7, Section 6.1.d(2). As a result of the review, RL concurs with the contractor's justifications presented in the deviation request.

The deviation request pertains to DOE 6430.1A, Section 1530-99.0, "Requirement for the Installation of an Automatic Fire Suppression System in a Nonreactor Nuclear Facility." The specific facility is the 241- - -91 Process Building installed under Project $1-320$. The 24l-C-9l Process Building is a 15 foot by 25 foot insulated, pre-engineered, metal building that contains equipment for air treatment. A fire event in the structure will not produce a Maximum Possible Fire Loss (MPFL) that would warrant the installation of an automatic fire suppression system. Also, radiological and toxicological releases are not postulated to occur as a result of MPFL event.

If any direction is provided by a Contracting Officer's Representative (COR) which your company belleves exceeds the COR's authority, you are to imediately notify the contracting officer and request clarification prior to complying with the direction.

If you have any questions, please contact Wahed Abdul on 372-2355.

WSO:WA

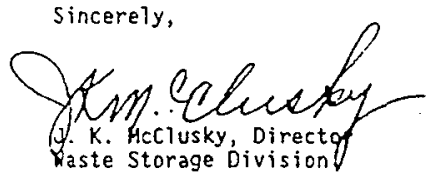

cc: H. M. Bueci, FDH

C. E. Hatch, FDH

A. M. Umek, FDH 